Cahiers Charlevoix

Études franco-ontariennes
Cahiers Charlevoix Études franco-ontariennes

or Crevenerix of

\title{
Le Principe du limaçon ou la résistance des marges. Essai d'interprétation de la dynamique des traditions
}

\section{Jean-Pierre Pichette}

Volume 8, 2010

URI : https://id.erudit.org/iderudit/1039317ar

DOI : https://doi.org/10.7202/1039317ar

Aller au sommaire du numéro

\section{Éditeur(s)}

Société Charlevoix

Presses de l’Université d’Ottawa

ISSN

1203-4371 (imprimé)

2371-6878 (numérique)

Découvrir la revue

Citer cet article

Pichette, J.-P. (2010). Le Principe du limaçon ou la résistance des marges. Essai d'interprétation de la dynamique des traditions. Cahiers Charlevoix, 8, 11-75. https://doi.org/10.7202/1039317ar
Résumé de l'article

Jean-Pierre Pichette reprend le fil de l'enquête qu'il a menée sur la sanction de l'aîné célibataire, une pratique qui a profondément marqué le rituel du mariage franco-ontarien. Considérant sa vitalité dans les régions périphériques, opposée à son déclin dans les zones centrales qui en suggère la continuité improbable, l'auteur confirme la résistance de la tradition dans les marges, phénomène qu'il avait auparavant observé en littérature orale. Dès lors, sur cette base élargie, il expose, en guise d'interprétation des dynamiques en présence, le " principe du limaçon ", un modèle tout simple capable d'apporter un éclairage original et significatif sur les rapports entre centre et périphérie, sur les possibles croisements ou l'origine plurielle des traditions ; il tente ainsi de cerner les facteurs de leur maintien ou de leur érosion en cherchant pourquoi les marges retiennent mieux et plus longtemps certains des messages transmis qui s'érodent au centre, et comment ce dernier en assure néanmoins la sauvegarde. Sans être universelle, cette proposition, que l'auteur livre comme réflexion finale à son enquête, se vérifie dans plusieurs cas de transfert culturel et elle appelle d'autres investigations afin d'en démonter le mécanisme complexe, délicat et capricieux. 


\title{
Le Principe du limaçon ou la résistance des marges. Essai d'interprétation de la dynamique des traditions
}

\author{
JeAn-Pierre Pichette \\ Chaire de recherche du Canada en oralité \\ des francophonies minoritaires d'Amérique (COFRAM) \\ Université Sainte-Anne, Pointe-de-l'Église
}

À la mémoire du père Germain Lemieux (1914-2008), ouvrier de la marge, décédé à Saint-Jérôme le 26 mars 2008 


\section{SOMMAIRE}

AvANT-PROPOS

I - LA RÉSISTANCE DES MARGES $\quad 20$

A. Le principe du limaçon 20

Centrifugation culturelle $\quad 21$

Concentration culturelle $\quad 22$

Exposition des dynamiques 23

B. La marge et ses attributs $\quad 25$

Les avatars de la marge $\quad 26$

Un conservatoire culturel 28

C. La marge, terrain idéal, terrain idéalisé 31

Les enjeux de la recherche 31

La leçon de Longfellow ou le regard de l'Autre 33

La "crotte de mouton » ou la honte de Soi 36

D. Causes et conditions de la résistance 38

Les causes $\quad 38$

Les conditions $\quad 39$

L'érosion de la marge $\quad 42$

Le complexe de la marge $\quad 45$

II - Autres TEMPS, AUTRES MARgES 47

A. Amérique française $\quad 47$

La « Guillonnée 》 au Missouri 47

La ceinture fléchée dans l'Ouest 48

La mi-carême en Acadie $\quad 50$

Les petits pains de sainte Geneviève à Québec $\quad 51$

B. Amérique anglaise $\quad 52$

La célébration de la Saint-Patrice 53

La gigue néo-écossaise 54

Les traditions terre-neuviennes $\quad 54$

Les chansons des Appalaches $\quad 55$

$\begin{array}{ll}\text { CONCLUSION } & 57\end{array}$

ANNEXE : REgARDS ET DISCOURS SUR LA MARGE 59

A. Des « Français intacts» $\quad 59$

B. La fascination d'un folklore vivifié 67 


\title{
Le Principe du limaçon ou la résistance des marges. Essai d'interprétation de la dynamique des traditions ${ }^{1}$
}

«Vous, vous venez d'un pays sans mémoire. »

Alain Minc ${ }^{2}$

«Au reste, la France est un peu oublieuse de sa nature... Nous, Canadiens, qui avons la mémoire un peu plus fidèle, venons à son secours; tâchons de lui rendre intact le précieux dépôt de ses anciennes chansons, comme nous pourrions, au besoin, lui remettre sous les yeux le tableau de ses moeurs et coutumes d'autrefois. 》 Hubert La RuE ${ }^{3}$

\begin{abstract}
Avant-propos
Étonné de découvrir en Ontario français un rituel du mariage qui n'avait encore retenu l'attention d'aucun chercheur, tant en ce pays qu'ailleurs au Canada, nous en avons fait l'objet d'une série d'enquêtes intensives conduites avec la participation de nombreux collaborateurs, collègues de partout, équipes d'étudiants de l'Université de Sudbury et, enfin, assistants de notre Laboratoire de littérature orale de l'Université Sainte-Anne. Ainsi, nous avons pu décrire, pour la première fois en milieu franco-ontarien, et
\end{abstract}

1. Cet article développe l'ultime volet d'une étude en cours depuis une dizaine d'années et dont nous avons livré les résultats dans les Cahiers Charlevoix. Études franco-ontariennes [désormais CC] (Sudbury, Société Charlevoix et Prise de parole) : J.-P. Pichette, «"Danser sur ses bas". Rémanence d'une sanction populaire dans le rituel du mariage franco-ontarien », CC, n 5, 2002, p. 229-311; et « De La Mégère apprivoisée au Roman de Julie Papineau. Origines d'un rituel du mariage francoontarien », $C C, \mathrm{n}^{\circ} 6,2004$, p. 195-248.

2. Alain Minc, économiste français, à Denise Bombardier, animatrice de l'émission « Raison Passion », Société Radio-Canada, télévisée le 28 janvier 2001 ; réplique de D. Bombardier : « Oui, mais nous partageons aussi la vôtre... »

3. Hubert La Rue, «Les Chansons populaires et historiques du Canada », dans Le Foyer canadien, Québec, tome I, 1863, p. 337-338. 
analyser par le menu la sanction de l'aîné célibataire dans ses formes présentes et passées : la très florissante « danse sur les bas » et la « danse dans l'auge » ou dans une cuve. Cette première exploration s'étendit graduellement à toute l'Amérique française, puisque le rituel était aussi corroboré, quoique de façon restreinte, au Québec, en Acadie et aux États-Unis ${ }^{4}$. La deuxième étape de cette recherche, suite naturelle de la première, nous engagea dans la quête de l'origine de ce comportement populaire, qui s'était révélé exclusif à la tradition française d'Amérique. Une nouvelle surprise se présenta alors : ce rituel, dont il existait pourtant de nombreuses configurations en France et dans la francophonie européenne, y était inconnu sous les formes pratiquées chez nous ; bien plus, c'est la filière britannique, dans ses éléments anciens, qui exposait les traces les plus claires d'une possible ascendance. Mais, déjà désuet en Angleterre et, de ce fait, généralement ignoré des Anglo-Américains, ce rite de passage, supposément d'origine celte, n'avait pas pu s'infiltrer sur le continent par la voie de la majorité anglophone. Selon l'hypothèse la plus vraisemblable, il aurait d'abord circulé et fleuri parmi les Celtes, les Bretons et les Français de l'Ouest, s'y serait généralisé avant de migrer en Canada avec les vagues de colons français venus s'y établir au $\mathrm{XVII}^{\mathrm{e}}$ siècle $^{5}$.

Ces faits admis, avec toutes les réserves que les hypothèses impliquent, c'est la vigueur de la tradition qui devait encore une fois piquer notre curiosité. La distribution géographique, présentée dans notre premier exposé, annonçait déjà ce phénomène : «En somme, ce qui frappe de prime abord, c'est la grande vitalité de ce rituel du mariage dans les régions périphériques du Québec (le Richelieu, l'Abitibi, le Témiscamingue et l'Outaouais), et dans les milieux minoritaires (l'Ontario et le Nouveau-Brunswick), en regard de son absence presque générale au cœur des régions du Centre (Québec, Trois-Rivières et Montréal) ${ }^{6}$.» L'examen des sources françaises conduisit au même étonnement : "Aucune

4. J.-P. Pichette, «"Danser sur ses bas " [...]», op. cit.

5. Id., « De La Mégère apprivoisée [...] », op. cit.

6. Id., «"Danser sur ses bas" [...]», op. cit., p. 297. 
attestation française, donc, de la danse de l'aîné nu-pieds ou en pieds de bas, dans une auge ou dans une cuve. Comment expliquer l'absence contemporaine de cette forme de la tradition en France, elle qui aurait dû en être le foyer de diffusion pour la francophonie nord-américaine ${ }^{7}$ ? » L'omission de ce rituel, tant dans les régions centrales du Québec que du côté de la mère patrie, trahirait-elle un phénomène d'érosion de la tradition?

Dans ce contexte, la question du transfert des traditions européennes au sein des populations de la francophonie nordaméricaine devint prioritaire et l'examen des échanges interculturels, pratiqués entre les groupes français eux-mêmes, puis entre ces derniers et les Amérindiens ou les Anglo-Américains, dans certains domaines des traditions orales, allait teinter de diverses manières les travaux et les articles qui en dérivèrent, quand ces sujets n'en formèrent pas tout bonnement le cœur ${ }^{8}$.

Cette interrogation nous ramenait directement à des observations antérieures. Notre communication synthèse de mai 1991, mesurant l'ampleur de « la diffusion du patrimoine oral des Français d'Amérique », démontrait en effet que les populations françaises du Canada avaient maintenu, souvent davantage et mieux que dans la France d'origine, un certain nombre de traditions orales 9 . Les données disponibles pour le conte et les jurons, deux genres familiers, permettaient de conclure que, ce faisant, elles avaient « en quelque sorte acquis le statut de "société

7. Id., « De La Mégère apprivoisée [...]», op. cit., p. 220-221.

8. La mise en place du projet « Entre la France et le Canada : la Bretagne » en 2000 et l'établissement en 2004 de la chaire COFRAM en découleront et mèneront à un colloque international sur "La Résistance des marges », qui a réuni une quarantaine d'intervenants à l'Université Sainte-Anne, Pointe-de-l'Église (Nouvelle-Écosse), du 15 au 18 août 2007. Les actes viennent de paraître : La Résistance des marges. Exploration, transfert et revitalisation des traditions populaires des francophonies d'Europe et d'Amérique. Actes du colloque international organisé à l'Université SainteAnne du 15 au 18 août 2007, sous la direction de J.-P. Pichette, Port-Acadie. Revue interdisciplinaire en études acadiennes, $\mathrm{n}^{\mathrm{os}} 13-14-15$, printemps 2008-automne 2008printemps 2009, $478 \mathrm{p}$.

9. Id., « La Diffusion du patrimoine oral des Français d'Amérique », dans Langue, espace, société. Les Variétés du français en Amérique du Nord, sous la direction de Claude Poirier avec la collaboration de Aurélien Boivin, Cécyle Trépanier et Claude Verreault, Sainte-Foy, Les Presses de l'Université Laval [PuL], « Culture française d'Amérique », 1994, p. [127]-143. 
distincte" » par l'originalité de leur production culturelle. Par exemple, pour une population environ sept fois moindre, les Français d'Amérique avaient fourni à la fin des années 1970 autant de contes en nombre absolu, environ dix mille, que toutes les provinces de la mère patrie réunies ${ }^{10}$. Pour illustrer leur statut distinct, nous référions ensuite à notre étude des jurons canadiensfrançais ${ }^{11}$, qui avait établi que les jurons religieux français s'étaient solidement maintenus à l'oral au Canada, principalement au Québec, où ils forment plus de $92 \%$ du corpus et se sont même renouvelés en « sacres », et qu'ils entretenaient de toute évidence la dimension religieuse des jurons de la vieille France. Nous traduisions ainsi cette originalité : «Par les multiples interjections de l'ancienne France qu'il garde toujours vivantes, le répertoire des jurons franco-canadiens manifeste une vitalité très française d'autant plus qu'il a su adapter les procédés traditionnels d'euphémisation à sa propre expérience culturelle. Il révèle aussi une créativité prodigieuse si l'on considère qu'un millier de termes, à peu près tous inconnus en France, s'emploient à la seule fin de tempérer moins d'une vingtaine de jurons [religieux] dits injurieux et que 539 d'entre eux sont des néologismes ${ }^{12}$. »

Une telle prédisposition pour le juron religieux tranche assurément sur la tendance actuelle en France. Ainsi, distinguant l'injure du juron, qu'il considère « en perte de vitesse », l'auteur d'un dictionnaire des injures s'interroge en note : « La décadence du juron est-elle une conséquence de la régression de l'esprit

10. Estimation française de Paul Delarue, Le Conte populaire français, Paris, Éditions G.-P. Maisonneuve et Larose, 1957 (1976), tome I, p. 34. Pour sa part, Luc Lacourcière évaluait à 9000 « le nombre total des versions de contes retrouvées dans la tradition orale française nord-américaine » à la fin des années 1960, dans Germain Lemieux, Placide-Eustache. Sources et parallèles du conte-type 938, Québec, PuL, 1970, « Préface », p. vIII. Compte tenu des enquêtes menées au Québec, en Ontario, en Acadie et en Louisiane durant la décennie 1970-1980, le cap de 10000 contes a été largement dépassé.

11. J.-P. Pichette, Le Guide raisonné des jurons : langue, littérature, histoire et dictionnaire des jurons, Montréal, Les Quinze, « Mémoires d'homme », 1980, 305 p.

12. Id., "Jurons franco-canadiens : typologie et évolution», dans Le Statut culturel du français au Québec, Actes du congrès Langue et société au Québec, tome II, Textes colligés et présentés par Michel Amyot, Québec, Éditeur officiel du Québec, 1984, p. 251. 
religieux $^{13}$ ? »; quelques pages plus loin toutefois, sa réponse tombe, catégorique : « le matérialisme sordide des masses a peu à peu fait tomber en désuétude tous les jurons sans distinction, qu'ils en appellent au Roi du Ciel ou au Prince des Ténèbres ${ }^{14}$ ». La parution tardive, mais toute récente, d'un premier dictionnaire des jurons franco-européens corrobore cette tendance en attestant que l'emploi du juron religieux est, de nos jours, perçu comme un archaïsme confiné au domaine littéraire et qu'il a cédé sa place à une trinité, toute profane celle-là, bordel, merde et putain $^{15}$.

Ainsi, ces travaux sur le conte et les jurons confirmaient que les traditions orales françaises ont bien survécu au choc de leur transplantation en Amérique et qu'elles ont gardé leurs traits caractéristiques; qu'elles se sont enrichies dans ce terreau nouveau et adaptées au contact des populations locales, et même, comme le cas des jurons le prouve hors de tout doute, qu'elles se sont renouvelées au cours des siècles. Parallèlement à cette rétention, le sort du patrimoine oral dans la société mère, la France, qui a doté sa colonie de contingents d'émigrants, se pose avec acuité si l'on considère seulement la danse de l'aîné célibataire.

Parce que cette facette a été peu étudiée, on peut facilement tenir pour acquis que la mère patrie a conservé ses traditions comme elle sait très bien le faire pour ses monuments architecturaux ; on pourrait en outre inférer, comme allant de soi, que l'absence d'une telle architecture moyenâgeuse en Amérique du Nord est en quelque sorte l'image d'une érosion culturelle causée par les migrations, étant donné qu'il est rationnel de penser que les migrants n'apportaient avec eux que le strict nécessaire. Ce sont là des apparences, trompeuses il nous semble, qui conduisent parfois à des positions aussi gratuites que celles de l'émissaire britannique lord Durham, considérant les Canadiens français

13. Robert Édouard, Les 9300 gros mots. Dictionnaire des injures de la langue française, [Paris], Tchou éditeur, [1967, 1979], p. 19.

14. Ibid., article « Bon Dieu!», p. 78.

15. Pierre Enckell, Dictionnaire des jurons, Préface de Jacques Réda, Paris, Presses Universitaires de France [PuF], 2004, 801 p. 
comme « un peuple sans histoire et sans littérature ${ }^{16}$ » ou, selon l'économiste français cité en exergue, Alain Minc, venant d'un « pays sans mémoire ». Pourtant ces remarques sans nuances sont contredites par les faits que livre l'étude des traditions populaires.

Comment pourrait-on décemment reprocher à ces étrangers leurs préjugés sans d'abord admettre les siens propres ? N'étaitce pas précisément la présomption que nous entretenions nousmême sur la grande perméabilité des minorités aux pressions assimilatrices majoritaires qui avait retardé l'examen de ce rituel inconnu dans nos terrains chez des groupes majoritaires ${ }^{17}$ ? Nous avions d'autre part déjà constaté, à propos de la transmission d'un conte français retrouvé chez les Amérindiens, que des arguments « qui relèvent davantage du contexte sociopolitique ou de préjugés bien alimentés par le monde de l'information », de nature autre que scientifique donc, bloquaient carrément la réflexion : par exemple, " l'idée d'une irrémédiable dégradation de la tradition orale des blancs », jointe à «l'ignorance de l'histoire » et au « manque de références culturelles » des populations minoritaires, empêchaient l'étude et conduisaient même parfois à nier l'évidence ${ }^{18}$.

Prôner la résistance de la marge dans une telle conjoncture paraît fort hasardeux tant cette position heurte de front certaines idées reçues. En effet, par quelle logique expliquer que des populations minoritaires et périphériques auraient mieux conservé leurs traditions que les majorités du centre quand des taux d'assimilation galopante, ratifiée par des études nombreuses et bien documentées, confirment régulièrement leur transfert vers la langue majoritaire et, conséquemment, l'abandon du véhicule de leur patrimoine oral ? Aussi, le point de vue de Gérard Bouchard, qui formulait l'hypothèse d' " une érosion de certaines

16. Lord Durham [John George Lambton, comte de Durham], Le Rapport Durham [1839]. Traduction [par] Denis Bertrand et Albert Desbiens. Introduction et appareil didactique [par] Denis Bertrand et André Lavallée, [Montréal], Les Éditions Sainte-Marie, "Cahiers Sainte-Marie », $\mathrm{n}^{\text {os }} 13-14,1969$, p. 123.

17. J.-P. Pichette, " "Danser sur ses bas " [...]», op. cit., p. 235.

18. Id., " "Le Lynx et le renard". Un relais déroutant dans la transmission du conte populaire français en Ontario », $C C, \mathrm{n}^{\circ} 1,1995$, p. 198 et 200-201. 
formes culturelles » en « contexte de peuplement ou de région neuve », paraît-il beaucoup plus raisonnable ${ }^{19}$ et justifierait sans peine la terminologie que ce chercheur emploie pour qualifier ce qu'il considère la simplification générale des coutumes du Saguenay et du Québec - « appauvrissement des vieilles pratiques coutumières », " régression des modes de vie », "grand dénuement », « déritualisation de la vie sociale » et « érosion des codes coutumiers » - en regard du vocabulaire réservé aux traditions plus élaborées - « foisonnement », « prolifération » et « rituels très nombreux et très raffinés »-qui aurait « caractérisé la plupart des sociétés rurales de l'Ouest français jusqu'au début du vingtième siècle ${ }^{20} »$. Toutefois, pour les exemples précités, comme maintenant pour la tradition de l'aîné célibataire, l'érosion n'est pas venue du côté qu'on l'attendait ${ }^{21}$.

19. Gérard Bouchard, « Sur la dynamique culturelle des régions de peuplement », The Canadian Historical Review, vol. LXVIII, n 4, 1986, p. 473-490. Voir aussi la mise à jour de ce projet: Gérard Bouchard, Josée Gauthier et Marie-Josée Huot, «Permanences et mutations dans l'histoire de la culture paysanne québécoise », dans La Construction d'une culture. Le Québec et l'Amérique française, sous la direction de Gérard Bouchard avec la collaboration de Serge Courville, Sainte-Foy, PuL, « Culture française d'Amérique », 1993, p. 261-305.

20. Ibid., p. 477. Ce vocabulaire sera assimilé par les membres de son équipe dans des articles et des thèses, notamment dans Dynamiques culturelles interrégionales au Québec et en France. Construction d'une enquête, sous la direction de Gérard Bouchard et Martine Segalen, Chicoutimi, Institut interuniversitaire de recherches sur les populations (IREP), 1995, [3]-260 p. Si Denise Girard évoque encore, avec prudence, la simplification dans son article « La Nuit de noces » (Cap-aux-Diamants, $\mathrm{n}^{\circ}$ 49, 1997, p. 19-21), Martine Tremblay se fait plus nuancée dans l'édition de sa thèse sur Le Mariage dans la vallée du Haut-Richelieu au XX siècle. Ritualité et distinction sociale (Sainte-Foy, Pul, « Géographie historique », 2001, XIII-294 p.).

21. Anne-Marie Desdouits, collaboratrice de l'équipe, produit pareille conclusion en présentant les premiers résultats de sa recherche : "Tout cela semble donc aller dans le sens de l'hypothèse d'une simplification des rituels », mais elle demeure prudente, comme l'indique cette note : « Il ne semble pas y avoir vraiment eu formation de nouveaux rituels, par innovation ou par emprunt, mais c'est la poursuite des recherches qui permettra de le confirmer ou, au contraire, de l'infirmer » (p. 327) : voir « Les Rituels du mariage paysan sur la Côte-de-Beaupré et dans la Beauce », dans La Construction d'une culture, op. cit., [307]-328. Cependant, des difficultés d'ordre méthodologique ont réduit l'hypothèse originale d' « une sorte de déperdition dans les formes rituelles venues de France » à des problématiques réformées et à des objectifs de comparaison plus modestes : voir Gérard Bouchard, Michelle Salitot, Martine Segalen, «La Structure des rituels matrimoniaux au Québec et en France au cours de la première moitié du $\mathrm{xx}^{\mathrm{e}}$ siècle ", dans Une langue, deux cultures. Rites et symboles en France et au Québec, sous la direction de Gérard Bouchard et Martine Segalen, [Québec], Pul, et [Paris], La Découverte, 1997, p. [13]-32. 


\section{I - LA RÉSISTANCE DES MARGES}

\section{A. Le principe du limaçon}

L'étonnante capacité de rétention du fonds traditionnel français - mise à jour ici pour l'Ontario par le foisonnement des formes et la vitalité de ce rituel du mariage ainsi que par sa présence attestée historiquement dans toutes les grandes régions de l'Amérique française - dévoile, par contrecoup, l'érosion du patrimoine culturel au sein même de la majorité, en cette «France oublieuse » comme l'avait qualifiée un siècle et demi plus tôt Hubert La Rue. La résistance culturelle des populations, qui ont migré, de gré ou de force, et qui se retrouvent en marge de leur culture d'origine ou, minoritaires, en marge aussi des cultures d'accueil, repose sur des conditions qu'il reste à préciser et répond à des situations sans doute variables d'une marge à l'autre, car, on peut le présumer, l'état minoritaire n'est pas partout uniforme. Or, pour comprendre comment certaines traces ont persisté dans les régions périphériques, cerner les facteurs qui favorisent leur maintien ou leur érosion, tant en périphérie qu'au centre, évaluer la performance des minorités acadiennes, franco-canadiennes et francoaméricaines à cet égard ainsi que leurs rapports avec les centres d'origine et les pôles d'attraction contemporains, et caractériser, pour mieux les décrypter, les mécanismes à l'œuvre dans ces milieux, c'est l'exploration de la littérature orale et des traditions qu'elle propage, dans des secteurs inaccessibles aux spécialistes du document écrit, qui devient pour nous la voie privilégiée. On ne peut éluder la question fondamentale que soulève cet état de fait : comment expliquer qu'une population périphérique ait conservé une tradition oubliée au centre et, conséquemment, qu'elle 1'ait fait dans des conditions adverses, malgré une marginalisation ou une migration et, encore, en situation minoritaire? Ce questionnement mène à une réflexion plus 
générale sur les rapports entre le centre et la périphérie ${ }^{22}$ - par exemple, entre Paris et les régions de la France, ou entre la France et le Canada français, ou entre Québec, la capitale du Québec, et Montréal, la métropole, ou entre le Québec et 1'Ontario ou l'Acadie -, et sur l'état de conservation - meilleur en périphérie qu'au centre -, ce qui expliquerait peut-être les trous dans la mémoire des majorités en regard des souvenirs probants des minorités excentriques. Le principe $d u$ limaçon, un modèle d'exégèse vers lequel nos recherches antérieures conduisent, pourrait apporter un éclairage original sur cette question de la résistance des marges.

\section{Centrifugation culturelle}

Si les édifices et les monuments du passé, ceux qui correspondent aux établissements primitifs, sont ordinairement situés au cœur d'une civilisation ou d'une société établie, dans les centres-villes des grandes capitales notamment, il en va autrement pour le patrimoine immatériel. Cette étude d'un rituel du mariage a montré que c'est plutôt à la périphérie que subsistent le plus longtemps un certain nombre d'anciennes pratiques culturelles, dont les traditions orales. Pour exalter la notoriété qu'elles revendiquent et qu'elles ne peuvent pas toujours soutenir, les vieilles cités, convoitées, sollicitées et investies par des promoteurs de tous ordres, se convertissent en lieux de mémoire ; proclamées trésors nationaux, elles sont admises au nombre des joyaux du patrimoine mondial. La reconnaissance et le prestige qu'elles en retirent favorisent la sauvegarde et la mise en valeur de leurs vestiges historiques à des fins diverses - culturelles, politiques, pédagogiques, économiques et touristiques - et attirent, dans leurs musées et centres d'interprétation, les élèves et les étudiants, les ensei-

22. Ce type de rapports a été traité sur le plan économique, notamment par Samir Amin (Le Développement inégal. Essai sur les formations sociales du capitalisme périphérique, Paris, Éditions de Minuit, 1973, 365 p.) et Immanuel Wallerstein (Comprendre le monde. Introduction à l'analyse des systèmes-monde, Paris, La Découverte, 2006, 173 p.) dont les travaux ont surtout mis en évidence les disparités entre les pays du Sud et ceux du Nord, et la dépendance des premiers, situés à la périphérie du système capitaliste mondial, des seconds, qui en composent le noyau. 
gnants et les journalistes, les mandataires et les électeurs, les chercheurs et les curieux, sans compter les nombreux touristes qui, tous à leur façon, désirent les visiter, pour admirer et connaître ces traces de civilisation, les voir donc et se faire voir en ces lieux consacrés par la culture de l'élite. Pour ces cures patrimoniales, interminables et combien ruineuses, les vieilles cités se délestent de leurs résidents encombrants, horde bigarrée de journaliers mal nantis et de petits artisans impécunieux qui ont fait leurs quartiers de ces édifices vétustes et bon marché, parce que délabrés ou abandonnés, et elles les repoussent à l'écart, dans les banlieues qui se développent autour. Des urbanistes réaménagent alors la coquille architecturale évidée pour y loger à grands frais de nouveaux habitants, mieux harmonisés avec la vocation rajeunie de la doctrine officielle ; les capitales, privées désormais de cette animation essentielle, demeurent la ruine d'un état révolu ; de milieux de vie et de création, elles sont devenues étalages factices, villes sans âme, mais rivales dans la course à l'ostentation patrimoniale.

Le monde animal possède des images propres à illustrer ce phénomène. Celle du limaçon ou de l'escargot, par exemple, qui aurait déserté sa carapace, abri transitoire, pour aller vivre ailleurs. Le gastéropode, qui l'a fabriquée, n'y habite plus ; un bernardl'hermite - crustacé parasite qui hante les coquillages inhabités y tient désormais résidence. Mais l'escargot vit encore. Dans les lisières où il s'est retranché, il a recréé un nouvel habitat et il continue là, dans la marge, à façonner selon l'usage ancien. Et c'est ainsi que le centre, agité par le tourbillon continuel des modes naissantes - qu'il découvre, attire, reproduit ou répand -, abandonne à la périphérie les formes périmées dont il se détourne. C'est la centrifugation culturelle.

\section{Concentration culturelle}

Toutefois, sur le plan de la conservation du patrimoine, la démarche est inversement proportionnelle au phénomène d'érosion. C'est le centre qui note cette éclipse et s'inquiète de 
l'illusion entretenue par la réputation des vieilles cités, qui découvre ce mouvement centrifuge, qui crée les institutions centres universitaires, musées, archives - nécessaires à sa récupération afin de se vouer à son étude et d'organiser sa préservation. Son intervention se compare ici à celle de l' " antiquaire »folkloriste, ethnographe, ethnologue des temps passés - : il repère la cantilène que l'interprète populaire chante, adapte et maintient ; il note l'ancienneté de sa mélodie, la simplicité de ses paroles et la naïveté de l'ensemble, et l'enregistre pour mieux la sauvegarder, l'analyser et la propager. Il est certain que son geste sera salutaire, car le témoin qu'il a entendu n'aurait pas eu de son propre chef l'idée de consigner sa poésie. En cela, l'antiquaire, qui se tourne vers la périphérie, dirige le bras tentaculaire de l'institution, puisque, en entreposant dans ses cartons, pour des fins de recherche et de mise en valeur, la tradition délaissée chez lui et qui a perduré ailleurs, il la soustrait à son milieu naturel, l'immobilise et la récupère en vue d'enrichir les archives nationales de la collectivité. Par ce ministère, il contribue à renverser la tendance et il produit peut-être ainsi l'illusion que c'est le centre qui a su garder la tradition vivante. Cependant, pour jouer son rôle, il a besoin de la mémoire du peuple qui, bourgeois-gentilhomme des temps nouveaux, détient la tradition, souvent sans le savoir ni en connaître la valeur. C'est la démarche culturelle inverse, entraînée cette fois par une action centripète.

\section{Exposition des dynamiques}

Cette métaphore, suffisamment féconde, apporte quelque lumière sur les caprices apparents de la transmission des pratiques et des usages au sein des peuples, tout en illustrant commodément l'action conjuguée des dynamiques qui les accompagnent. Parmi celles-ci, la première serait certes la tonicité de la tradition, soit sa vigueur, qui se remarque a priori par la grande stabilité de ses composantes; grâce à cette force, les observateurs et les chercheurs étrangers ont pu reconnaître la vitalité et la fidélité, qu'ils ont souvent qualifiées d'incomparables, des traditions 
françaises au Canada, notant particulièrement le maintien des traits les plus archaïques ${ }^{23}$. En ce qui concerne la sanction de l'aîné célibataire, dont l'examen des pérégrinations est le point de départ de cette réflexion, elle en expose les lieux de résistance fondamentaux ; sa constance a permis de décrire la tradition vivante du rituel partout en Ontario, et ailleurs où il a été signalé, et même d'envisager son évolution. Une deuxième force, dynamique corollaire, y est associée : la faculté de s'intégrer à des sociétés, des cultures, des contrées et des époques diverses, et d'y persister. Cette adaptabilité est bien évidente dans le déplacement de la coutume sous enquête : vraisemblablement courante chez les Celtes anciens, elle se serait répandue, à partir d'un lieu qui demeure inconnu, dans les Îles britanniques et aurait aussi atteint la Bretagne ; par là, elle se serait introduite dans une partie de la francophonie avant de migrer en Amérique. Si la dynamique de la diffusion est notoire, convenons que le cheminement mis à jour par notre étude paraît bien singulier et, précisément parce qu'il apparaît à son terme, qu'il n'était guère concevable dans une problématique qu'on aurait avancée au départ.

À l'inverse et sur les deux continents, l'Europe et l'Amérique, une troisième dynamique, négative celle-là, aurait opéré en parallèle dans le processus de transmission : la caducité de la tradition. Évoqué par Shakespeare au $\mathrm{XVI}^{\mathrm{e}}$ siècle, ce rituel du mariage se serait éteint en Angleterre sans laisser d'autres traces que les brèves descriptions figurant au terrain des folkloristes du $\mathrm{XIX}^{\mathrm{e}}$ siècle. Plus encore, en France, la coutume supposément connue des Bretons se serait ensuite généralisée dans la francophonie de l'Ouest sans même avoir jamais été décrite par des folkloristes français. Nous déduisons ce fait de la popularité de la tradition répandue par les Français à la grandeur du continent nord-américain. D'autre part, cette dernière constatation, qui montre la lacune européenne, révèle maintenant une lacune

23. L'annexe « Regards et discours sur la marge » regroupe une série de tels témoignages qui montrent la continuité de ces perceptions. 
similaire au Québec, le cœur de la francophonie du Nouveau Monde. En somme, l'érosion a agi au sein même des centres de diffusion.

Ainsi, tout cela est rendu perceptible, et donc vérifiable, par une quatrième dynamique : le maintien de la coutume dans les zones marginales ou satellites des centres. Partout où nous l'avons trouvée, elle est le fait d'une population située à la périphérie ou minoritaire ; c'est le cas de la francophonie dans toutes les provinces du Canada et aux États-Unis. De plus, ces minorités sont aussi des populations qui ont migré : de la France au Canada au temps de la Nouvelle-France ; puis de là, par des déplacements libres ou forcés au cours des siècles, vers l'Ouest canadien et les États-Unis. Le principe du limaçon peut s'ouvrir encore à des dynamiques accessoires - la dépendance des marges ou l'hégémonie des centres par exemple - et opérer à échelle réduite au sein du centre ou de la marge, dans les rapports entre individus et groupes notamment.

\section{B. La marge et ses attributs}

À l'instar de son prototype matériel, littéralement « l'espace blanc autour d'une page de texte écrit ou imprimé ${ }^{24} »$, la marge symbolise la périphérie, une zone ceinturant un noyau principal, dont elle constitue le seuil ou le terme. Ce positionnement spatial, forcément à l'écart du foyer et parfois même au-delà de ses limites, s'accompagne aussi d'un intervalle temporel, distance réelle entre le centre et la marge, qui s'appréciera diversement selon les points de vue adoptés oscillant, avec plus ou moins de nuances, entre ces extrêmes : la perspective méprisante qui déprécie la marge et l'accuse d'un retard indéniable sur le centre, tel un atavisme pénalisant systématiquement une condition jugée arriérée ; ou la perspective idéalisante qui magnifie au contraire cette situation comme un sursis stimulant la réflexion, sorte de prime à un état bienheureux. Bill Laws a bien rendu ce tiraillement, reflet de la

24. Le Nouveau Petit Robert, $40^{\mathrm{e}}$ édition, Paris, Dictionnaires Le Robert, 2007 [2006], p. 1537. 
« morgue des citadins à l'égard de leurs cousins des champs », dans le paradoxe : " les campagnards, s'ils ne sont pas faibles d'esprit, ont en tout cas l'esprit lent, mais ils vivent dans un paradis pastoral »; c'est là un préjugé qu'il anéantit aisément : «Le raisonnement métropolitain ne tient pas compte du fait que[,] si la seconde hypothèse était vraie, la première devrait nécessairement être fausse - si les ruraux étaient si stupides, comment eussent-ils pu découvrir le nirvâna ${ }^{25}$ ? »

Car les rapports entre centre et périphérie sont des arènes où s'affrontent de nombreux adversaires : l'indigène et l'étranger, le supérieur et l'inférieur, l'essentiel et l'accidentel, le général et le particulier, le sacré et le profane, la rectitude et l'erreur, le savant et le populaire, la ville et la campagne, la nouveauté et la tradition, l'écrit et l'oral, la norme et l'exception. Le décalage entre ces positions est tel que la réception d'un signe, selon qu'il est issu du centre ou de la périphérie, alourdit encore le contentieux. Bien sûr, fort de la supériorité que lui confère le nombre, le centre reste le pôle d'attraction. La marge, chargée d'une différence fondée sur la singularité, se résigne à la relégation, par choix ou de fait, ce qui implique qu'elle sera de peu de conséquence sur la majorité. Ainsi, est marginal ce qui est excentré, lointain ou isolé, minoritaire, hors norme, donc accessoire, secondaire, voire invisible.

\section{Les avatars de la marge}

Or, la marge est multiple. Liée au territoire et à la géographie, elle est palpable. Ce sont les régions par rapport au centre, la banlieue en regard du centre-ville, telle la basse ville de Québec comparée à sa haute ville, ou la ceinture de Montréal confrontée aux arrondissements urbains ; ce sont aussi les clivages entre les quartiers populaires d'un centre-ville et ses isolats cossus, l'écart entre le quartier ouvrier de Saint-Sauveur et le secteur huppé de

25. Bill Laws, Maisons \& demeures traditionnelles de la campagne française, [Paris], Éditions Albin Michel, 1995, [Traduction de Traditional houses of rural France, 1991], p. 13-14. 
Sillery, à Québec, ou le Moulin-à-Fleur et le « haut-de-la-côte » aisé de la rue Brébeuf, à Sudbury ; les rivalités entre la province et la capitale, comme l'antagonisme persistant entre Québec, la capitale « village », et Montréal, la métropole ; la distance entre la campagne et la ville, la marche ou la frontière opposée au siège de l'État, les colonies à la mère patrie.

Liée à la personne, la marge sera d'abord physique et ses distinctions basées sur l'anatomie ou les caractères biologiques : la race, l'ethnie, la couleur de la peau, les cheveux, le sexe, le quotient intellectuel, la latéralité - gauche ou droite -, la santé, la taille, le poids, etc. Puis elle se traduira dans les traits culturels d'un groupe : la science ou le degré d'avancement technique d'une civilisation, le niveau d'instruction des individus évoluant entre tradition et scolarisation; la religion et ses prescriptions, l'appartenance obligatoire ou facultative, la pratique régulière ou occasionnelle ; la langue utilisée et la manière de la parler avec ses accents, ses intonations, son vocabulaire, etc.

Toutes ces différences génèrent en outre des marges sociales, culturelles et économiques qui se traduisent par des relations de promotion ou d'exclusion. Sociale, la marge repose sur la place occupée dans le groupe, la classe dominante ou la classe populaire, fondée sur la naissance, le prestige de la propriété ou de la richesse, l'instruction ou le développement intellectuel, le nombre ou la minorité, la condition qui s'y rattache ${ }^{26}$, la considération de l'emploi occupé et le revenu qu'il procure, l'âge et l'expérience, ou l'origine. Culturelle, la marge séparera les natifs des nouveaux venus $^{27}$, elle fera des distinctions selon les habitudes alimen-

26. Par exemple, les Canadiens français perçus comme les Nègres blancs d'Amérique, les Chinois de l'Est ou les grenouilles, Frogs.

27. Comme dans cette blague entendue au Québec : «Il y a deux sortes de Français : des Français comme nous autres » - c'est-à-dire d'un abord simple, sympathiques, cordiaux -, « et des Français comme les autres »-c'est-à-dire hautains, prétentieux, arrogants. 
taire $^{28}$, le costume, etc. Politique, elle démarquera les camps les fédéralistes et les souverainistes, les conservateurs et les progressistes, les protectionnistes et les libre-échangistes -. Encore, elle fera la part entre l'exceptionnel, la vedette qui s'agite sous les réflecteurs, et l'ordinaire, la majorité silencieuse qui vit dans l'ombre. Enfin, la marge est mobile, car on enregistre des déplacements dans la marginalité ou encore de la marge au centre sous certaines conditions, qui prennent tantôt la forme de rites de passage positifs, comme l'obtention d'un diplôme ou d'une promotion, la réception d'un prix ou d'un honneur, tantôt, inversement, négatifs, comme la destitution d'une charge, le licenciement, la faillite ou le rejet.

Véritable multiplicité des points de vue, en effet, qui, soumis à un réseau complexe et mouvant de traits souvent subjectifs, balisent les relations d'individus coalisés sous la bannière d'un « nous » majoritaire ou celle d'un « eux » marginal. Fondée ici sur des critères mesurables, là sur des perceptions floues, la marge, notion toute relative, reste ainsi subordonnée aux humeurs du parti qui s'érige en centre et la définit. Ce que transpose à sa façon le refrain de la chanson : « On est toujours un peu l'Iroquois de quelqu'un / Que 1'on soit Québécois, Breton, Nègre ou Cajun / Je vous laisse à chanter quel peut être le vôtre / On est toujours un peu l'Indigène d'un autre ${ }^{29}$. »

\section{Un conservatoire culturel}

Excentrée, puisqu'elle est l'aboutissement d'une mode ou d'un fait qu'elle accueille tardivement, la marge se distingue comme le refuge naturel de traits de jadis, leur vivant conservatoire, voire

28. On a surnommé Mushrats French les Franco-Américains du Michigan parce qu'ils mangent du rat musqué [muskrat]; cette expression s'applique aux « deux côtés de la frontière » et touche donc la région de Windsor, selon Marcel Bénéteau et Peter W. Halford, Mots choisis. Trois cents ans de francophonie au Détroit du lac Érié, [Ottawa], Presses de l'Université d'Ottawa, « Amérique française », [2008], p. 8. De même, la soupe aux pois a valu aux Canadiens français l'appellation de Pea Soupers, façon désobligeante de désigner un Canadien français, spécialement du Québec, dans le parler familier des Anglo-Canadiens : $c f$. [wikipedia.org/wiki/pea_soup].

29. « Le Chanteur indigène », paroles et musique de Sylvain Lelièvre, Éditions Basse-Ville, 1977. 
le laboratoire d'une activité résistante. Les exemples abondent pour illustrer cette particularité, tant sur les plans physique et physiologique que culturel. La topographie conserve ainsi le relief d'une occupation ancienne, comme les débris d'une faïence extraite de fouilles récentes gardent, même après plusieurs siècles, les indices de son origine, perceptibles par la terre utilisée, sa forme, son émail, son dessin, ses couleurs et la marque de son fabricant, et un meuble divulgue son ancienneté et sa provenance par ses lignes, ses moulures, sa patine, les âges de son bois. De même qu'une cicatrice atteste chez un vieillard une blessure de jeunesse ou que la peau d'un jeune noir, descendant d'immigrants établis depuis des générations, reproduit les traits particuliers de ses ancêtres et l'assimile encore à un immigrant de fraîche date, le sang et l'ADN d'un individu constituent des traces génétiques qui l'identifient et certifient son ascendance ou sa descendance.

Les traces immatérielles ne manquent pas non plus. Le grasseyement d'un Québécois, le roulement des « $\mathrm{r}$ » d'un Montréalais ou l'intonation d'un Saguenayen trahissent l'origine d'un individu par cette signature régionale tout autant que l'argot d'un fonctionnaire, la diction d'un comédien, la langue châtiée d'un professeur ou le parler gras d'un manœuvre annoncent la qualité de leur formation et les situent dans l'échelle sociale : « Rien que par sa voix, j'ai bien vu qu'il sonnait instruit », déclara une réceptionniste sudburoise qui avait détecté que son interlocuteur était le conférencier attendu. À la langue de bois du politicien et au discours policé du centre, la marge oppose ses opinions franches et tranchées, ses réactions brutes parfois, comme ces « Normes de vie », édictées par la municipalité de Hérouxville $^{30}$, qui devaient défrayer les manchettes en janvier et février 2007 et contribuer d'une certaine façon à la formation de

30. La municipalité de Hérouxville, constituée en 1904, compte 1323 personnes et fait partie de la MrC Mékinac, comté de Laviolette, au Québec ; voir le portail [municipalite.herouxville.qc.ca/normes]. 
la Commission de consultation sur les pratiques d'accommodement reliées aux différences culturelles ${ }^{31}$.

En plus de l'élocution, le lexique régional permet de déterminer la provenance des habitants d'un pays : parlant d'une bouilloire, un Acadien emploiera généralement le mot «coquemar », un Québécois de 1'Est utilisera plutôt « bombe », et un Montréalais ou un Franco-Ontarien dira " canard»; aussi, un « trente-sous » désigne au Québec, en Ontario et dans l'Ouest du pays une pièce de vingt-cinq cents qui s'appelle un «quinzesous » chez les Acadiens de la baie Sainte-Marie. Ces derniers préfèrent toujours l'adverbe « point » à « pas » dans la négation et ils emploient couramment le passé simple avec finale en -i (-is, -it, -irent) ou en -u (-us, -ut, -urent) et l'imparfait du subjonctif, temps devenus ailleurs archaïques ${ }^{32}$.

Un peu partout au Canada français, on a relevé des expressions qui subsistent à une réalité disparue : en Ontario, on calcule encore la performance de son automobile en « milles au gallon », alors que, depuis plus de vingt-cinq ans, l'essence se vend au litre et la vitesse se mesure en kilomètres; au Québec, même si le système métrique prévaut depuis des décennies à l'école, on élève toujours des cloisons en « deux-par-quatre », colombages qui mesurent vraiment deux « pouces » par quatre « pouces », on achète encore parfois sa « pinte » de lait (contenant exactement un litre) et son « gallon » de peinture (quatre litres), ou sa « livre » de beurre (454 grammes), comme on le fait encore en France et en Belgique pour le demi-kilo (500 grammes). Par ailleurs, au Canada français, en Belgique, en Suisse et dans plusieurs régions de France, on a maintenu l'ancienne distinction « déjeuner », « dîner », « souper » plutôt que petit-déjeuner, déjeuner, dîner. Que dire en outre de la survivance de l'ordalie moyenâgeuse dans la protestation « j'en mettrais ma main au feu » ou du souvenir au moins bicentenaire de la déportation des Acadiens qui perdure dans « un cœur

31. Commission québécoise instituée en février 2007 : voir le portail [www.accommodements.qc.ca].

32. Philip Comeau, doctorant à l'Université York, confirme cet emploi des temps en français acadien. 
d'Anglais », appellation désignant une pomme de terre au cœur noir, mais blanche d'apparence ? Ces traits linguistiques négligés par le centre majoritaire, recueillis par un bon entendeur dans le discours marginal de la géographie ou de l'histoire, livrent souvent mieux que des signes officiels le parcours d'un individu ou d'une collectivité. Ces rogatons, qui conservent parfois leur perle comme l'huître pour qui sait l'ouvrir, constituent néanmoins des discriminants très nets qui, selon l'environnement où le locuteur intervient, le rangent du côté du centre ou de la marge.

\section{La marge, terrain idéal, terrain idéalisé Les enjeux de la recherche}

Si le principe du limaçon dit peu sur les causes des phénomènes observés, il éclaire les enjeux de la recherche ethnologique, discerne la part de la marge pour la vitalité des traditions de celle du centre pour leur protection, et il explique en outre la démarche des acteurs en présence - informateurs, enquêteurs et institutions. Il confirme que les premiers ethnologues, appelés folkloristes, ont eu raison de mener l'essentiel de leurs enquêtes en périphérie. Leur prédilection pour les milieux situés à la marge plutôt qu'au centre ne serait pas inspirée par un obscur appétit d'exotisme, mais correspondrait bien à la réalité des faits. Intuitivement, ils avaient compris qu'à l'écart du bouillonnement des villes, ils retrouveraient dans ces marches que composaient les collectivités rurales, comme l'écume rejetée avec les galets sur la grève, le fertile goémon que la tradition y avait déposé. Pour eux, toute marge est un terrain d'enquête idéal ; c'est pourquoi, elle est tellement idéalisée. Telle fut en effet l'expérience régionale et excentrique des folkloristes du XIX ${ }^{\mathrm{e}}$ siècle : Hubert La Rue puis Ernest Gagnon, qui dévoilaient des traditions chantées en marge de la France tout autant que de la bonne société de Québec. Ce fut celle aussi de la première génération d'ethnologues d'ici : de Marius Barbeau en sa Beauce natale comme en Charlevoix ou dans le Bas-Saint-Laurent, de Luc Lacourcière dans l'Acadie du Nouveau-Brunswick ou de l'Île-du-Prince-Édouard, provinces en 
marge du Canada central et urbain; de Joseph-Médard Carrière dans les anciens postes français des États-Unis et du Canada, spécialement au Missouri et dans la région du Détroit, d'Élizabeth Brandon en Louisiane, puis de Germain Lemieux dans le NouvelOntario, de Carmen Roy en Gaspésie ou dans l'Ouest canadien, d'Anselme Chiasson en Nouvelle-Écosse ou aux Îles-de-laMadeleine. Que dire encore de Geneviève Massignon en Acadie et des autres Français qui ont sillonné le territoire depuis Julien Tiersot jusqu'à Ariane de Félice ? De nombreux contemporains les ont suivis dans cette voie : Jean-Claude Dupont en Acadie et en Beauce, Roger Paradis dans le Maine, Gerald Thomas à TerreNeuve, puis Georges Arsenault à l'Île-du-Prince-Édouard, Barry Ancelet en Louisiane, Marcel Bénéteau dans le sud-ouest de l'Ontario, et d'autres encore.

Il est clair que la perspective d'œuvrer en terrain neuf et d'appréhender un patrimoine encore vierge, la fierté d'être du petit nombre d'experts à l'explorer, d'autant si la communauté est minuscule, inaccessible ou fragile, et, par là, de contribuer au progrès de la science, sont des dispositions à prendre en compte dans la motivation des chercheurs. D'autres petits bénéfices personnels ajoutent parfois de l'attrait à la marge : le privilège d'interroger les aînés, des informateurs susceptibles de disparaître les premiers, d'avoir peut-être ainsi côtoyé le dernier témoin vivant et enregistré ce que personne d'autre ne pourra ensuite observer, ou encore de refaire le parcours des pionniers et de consigner ce qu'ils n'ont jamais pu dénicher ni même soupçonner. Ainsi se vérifie un principe inspiré de l'économie : la rareté crée la cherté.

On pourra objecter que les devanciers se dirigeraient d'après les doctrines nationalistes et religieuses de leur époque - comme l'idéologie du bonheur rural de la thèse agriculturiste, dont les raccourcis « Restons chez nous » ou « Un royaume vous attend » étaient en parfaite symbiose avec la mission providentielle des Canadiens français, qui concevait « la langue gardienne de la foi et la foi gardienne de la langue $»-$. Pourtant, les succès de leurs 
terrains montrent qu'ils avaient visé juste et on voit mal comment on pourrait franchement démontrer qu'ils auraient accompli davantage dans les centres. Si tel avait été le cas - que le centre eût été le lieu idéal du maintien de la tradition -, on comprendrait mal que des spécialistes avertis du centre aient tant voulu s'astreindre aux nombreuses tracasseries - éloignement, adaptation, perte de temps - et aux frais supplémentaires - transport, hébergement et pension - qu'entraînait l'enquête en périphérie.

Il est vrai que l'ethnologue allant à la quête des traditions en milieu éloigné, excentrique, a communément tendance à accorder à cette marge l'exclusivité du maintien de la tradition dans toute son authenticité ou du moins dans ce qu'il tient pour son état le plus primitif. Une telle attitude entretient l'impression navrante que le centre n'est porteur d'aucune tradition digne d'intérêt. Pourtant, la ville n'est pas exempte de traditions; elle est même au contraire cousue de marges parfois plus subtiles qu'il faut également savoir décrypter : un patrimoine vivant, on le sait, est aussi plus difficile à identifier. Mais, là encore, c'est souvent à l'écart des modes vantées par les élites ou valorisées par la publicité qu'il gîte. Car la ville possède ses quartiers populaires, opposés à ses milieux dits branchés. Pourquoi s'y attarder, auraient pu penser certains des premiers folkloristes, quand on trouve des filons inépuisables en périphérie ? C'est néanmoins dans ces marges urbaines de Montréal qu'Édouard-Zotique Massicotte a recueilli ses chansons, croyances et dictons, et, comme Conrad Laforte et d'autres l'ont vérifié, qu'Ernest Gagnon avait noté, autour de lui à Québec, ses Chansons populaires du Canada. Le folklore urbain n'est donc pas une invention des années 1970.

\section{La leçon de Longfellow ou le regard de l'Autre}

En définitive, que les chercheurs soient allés ici ou là importe peu. Il leur suffisait simplement d'observer de l'extérieur, car le grand révélateur, c'est en définitive le regard de l'Autre. N'estce pas l'Autre, par sa distance et son questionnement, qui peut le mieux voir les caractéristiques d'une localité et lui renvoyer 
l'image qu'elle projette ? Ce qui semble vrai dans tous les cas et que l'autochtone admet avec réticence -, c'est qu'il est plus aisé à un observateur étranger à la marge de repérer la couleur locale d'une tradition, d'en saisir au premier abord la différence, qu'au natif pour qui elle est tellement familière qu'il ne la remarque plus. À l'inverse, il est aussi avéré que l'enquêteur de l'intérieur, désormais conscient de cette différence ou qui a expérimenté cette distance hors de son milieu, réussira mieux dans la cueillette des données que l'étranger qui n'a pas l'habitude et l'intimité que procure la longue fréquentation du milieu ${ }^{33}$.

Néanmoins, l'attention que porte le centre ou l'étranger à la marge déclenche chez celle-ci, par réaction, une prise de conscience qui la stimule à protéger plus efficacement son patrimoine. Le pouvoir de l'étranger, même s'il n'intervient qu'en spectateur, est bien réel. Par exemple, l'intérêt que des enquêteurs manifestèrent pour une laiterie de ferme en Ontario - dont ils avaient relevé, mesuré et photographié les particularités, puis interrogé des témoins pour en dater la construction et documenter les usages - a ravivé la fierté de son propriétaire qui, après leur passage, l'a rénovée et repeinte. Parfois, l'impact sera colossal, déterminant même l'orientation d'une carrière et ouvrant un champ de recherche. La question de l'anthropologue germanoaméricain Franz Boas, «Y a-t-il au Canada des contes anciens, comme les contes de fées d'autrefois ? », posée en 1914 à un Marius Barbeau ahuri, est de cet ordre. Sa portée, en conviendra ce dernier, « devait mouler une partie de [s]on avenir » et le lancer sur une piste imprévue, qu'il avait d'avance reniée, la jugeant sans intérêt: " Mais à Lorette, pendant mon relevé ethnographique, je me rappelai que Prudent Sioui et sa femme avaient commencé quelques-uns de ces contes, que j'avais refusés parce

33. Plusieurs des enquêteurs cités supra sont d'ailleurs issus des marges où ils ont œuvré : Barbeau en Beauce, Lemieux en Gaspésie, Chiasson à l'Île-du-Cap-Breton, Paradis dans le Maine, Arsenault à l'Île-du-Prince-Édouard, Ancelet en Louisiane, Bénéteau au Détroit. 
qu'ils étaient trop français et d'apparence trop littéraire ${ }^{34}$.» On peut interpréter comme décisive l'influence de ce savant étranger sur le développement de l'ethnologie au Canada français : les centaines de contes que Barbeau nota à la sténographie dans les quelques années qui suivirent, et dont il publia un nombre important dans des revues scientifiques et des éditions populaires, sont à l'origine des grandes collectes et des études comparatives de Luc Lacourcière et de ses étudiants de l'Université Laval. On a avancé encore que c'est le poème Évangéline, fruit de la vision romantique de l'Anglo-Américain Henry Wadsworth Longfellow, qui attira l'attention du monde sur le peuple acadien et marqua « l'éveil de la conscience collective des Acadiens », qui s'y étaient reconnus ${ }^{35}$.

En revanche, la marge se mobilise parfois contre les spoliateurs et les visiteurs qui l'auraient décrite de l'extérieur, sans une connaissance intime du milieu. Le regard du touriste sensible à la beauté et à l'originalité d'un objet artisanal, qui en tire des clichés, en achète des reproductions en souvenir et fait tourner l'économie locale, paraît moins menaçant que celui de l'universitaire qui le recueille pour des fins d'étude ou du brocanteur pour des intérêts mercantiles. Bien que ces visiteurs intéressés n'en contribuent pas moins à la vogue du patrimoine et à l'éveil de la conscience locale, seul un autochtone, aux yeux de la marge, peut bien comprendre et interpréter son patrimoine ; c'est pourquoi elle remet en cause l'idéologie, la méthode et jusqu'à l'éthique de ces étrangers, ou, lieux communs pour les ethnologues de terrain à qui l'on a ressassé de telles méprises ou trahisons, elle s'inquiète, se tait, se dérobe. Appréhendant semblable embarras, Georgiana Audet de l'île d'Orléans ferma sa porte aux chercheurs

34. Marius Barbeau, «En quête de connaissances anthropologiques et folkloriques dans l'Amérique du Nord depuis 1911 », Résumé d'un cours donné à la faculté des Lettres, Université Laval, mars-octobre 1945, p. 8.

35. Léon Thériault, "L'Acadie de 1763 à 1990, synthèse historique », dans L'Acadie des Maritimes. Études thématiques des débuts à nos jours, sous la direction de Jean Daigle, Moncton, Université de Moncton, Chaire d'études acadiennes, 1993, p. 55. 
étrangers à son île et organisa ses propres soirées de danses traditionnelles pour les sauver de l'oubli.

\section{La " crotte de mouton" ou la honte de Soi}

La démarche de lettrés mettant la périphérie au cœur de leurs recherches n'allait pas de soi non plus. Elle était originale, voire très marginale. Le parti pris de l'ethnologue de considérer, « du fait même de leurs connaissances particulières "), les gens du peuple comme « les supérieurs de l'étranger, même du savant, qui les recherchent, cahier et crayon en mains ${ }^{36} »$, était certainement une attitude révolutionnaire dans la première moitié $\mathrm{du} \mathrm{Xx}^{\mathrm{e}}$ siècle ; et, de plus, considérer qu'un " chanteur de chansons populaires[,] qui a quelque instruction de la ville, n'est pas d'ordinaire le meilleur » et que c'est « économiser le temps que d'avoir affaire à des gens de la campagne, dont les connaissances ne sont pas aussi confuses et mêlées », ne pouvait qu'engendrer de l'opposition ${ }^{37}$. Sur le terrain même, l'incompréhension de l'élite locale pouvait même nuire au travail des chercheurs. Le curé de Port-Daniel, en Gaspésie, qui avait assisté, et apparemment pris plaisir, à une séance de collectage, au cours de laquelle Marius Barbeau avait enregistré des « chansons de seigneurs galants et de bergères accueillantes », fustigea, du haut de la chaire et « dans un vert sermon », ces chanteurs de chansons qu'il condamna comme $«$ grivoises $»^{38}$.

Dans l'esprit des autorités qui s'activaient à relever le niveau d'instruction du peuple, cette direction intellectuelle était pure hérésie, tant il était alors inconcevable de se mettre à l'écoute de l'habitant, d'accorder de l'intérêt au discours de l'illettré, d'ériger ainsi l'ignorance en objet d'étude. Aussi, la réaction des milieux professionnels de l'entourage des Barbeau, Lacourcière et Lemieux fut-elle d'abord hostile. Luc Lacourcière essuya un jour la charge vexante d'un certain abbé, professeur à l'Université Laval, opposé à l'enseignement du folklore : «Nous, nous

36. Marius Barbeau, « En quête de connaissances [...]», op. cit., p. 41-42.

37. Ibid., p. 33.

38. Ibid., p. 37. 
essayons de sortir les étudiants de la campagne et, vous, vous les replongez dans leur crotte de mouton ${ }^{39}$ ! » Combien de fois Germain Lemieux n'a-t-il pas raconté à son tour les remarques d'un confrère jésuite qui, trouvant louches ses sorties le soir, l'avait dénoncé à ses supérieurs, et surtout de cet autre qui disait à tout venant que ce folkloriste avait passé sa vie à s'amuser à des niaiseries ${ }^{40}$. N'était-ce pas la réédition à trois siècles d'intervalle des critiques qu'avaient adressées à Charles Perrault « quelques personnes qui affectent de paraître graves » en « regard[ant] avec mépris » ces « bagatelles » de contes ${ }^{41}$ ?

On peut déduire de ces rebuffades que l'intérêt pour ce type d'études régionales était encore bien mince en ce $\mathrm{xx}^{\mathrm{e}}$ siècle des pionniers et que leur reconnaissance ne viendrait que beaucoup plus tard : le programme en études québécoises, dites au départ « canadiennes », fut l'un des premiers au milieu des années 1960 à l'Université Laval, et il doit beaucoup au mouvement de l'ethnologie inauguré par l'établissement de la chaire de folklore confiée à Luc Lacourcière en 1944 ; de même, les études francoontariennes naquirent une dizaine d'années plus tard à l'Université d'Ottawa sous l'impulsion du Québécois René Dionne, et les études acadiennes commencent tout juste à reprendre de la vigueur à l'Université Sainte-Anne, après quelques années de latence, alors que, près d'un demi-siècle après sa fondation, l'Université de Moncton en est toujours à l'annonce d'un programme d'enseignement de deuxième cycle ${ }^{42}$.

39. Ce à quoi Luc Lacourcière avait répondu : « Si c'est là la considération que vous avez pour le folklore, je vous la relance à la figure ». Anecdote citée de mémoire.

40. Dernière mention dans « Des honneurs pour le père Germain Lemieux : le folklore, source de la culture. Une entrevue menée par Pierre Bélanger, s.j. », s.d. [2006], publiée dans le portail des jésuites du Canada français, [www.jesuites.org/repertoire/ GermainLemieux] ; une version brève a aussi paru dans Compagnons, revue interne à la Province jésuite du Canada français et d'Haïti, vol. 25, n 3, automne 2006.

41. Charles Perrault, Contes de ma mère l'Oye. Illustrations de Gustave Doré, [Paris], Gallimard, «Folio junior », 28, [1697, 1977], p. 11 et 14. L'auteur justifia après coup son intérêt par la « morale louable et instructive » des contes : « Cela devrait me suffire pour ne pas craindre le reproche de m'être amusé à des choses frivoles. »

42. Cette université dispose depuis un bon moment d'un centre (1968; devenu Centre d'études acadiennes Anselme-Chiasson en 2008)) et d'une chaire d'études acadiennes (1982), d'un musée acadien (installé à la cité universitaire de Moncton en 
Même de nos jours, sur le terrain, les ethnologues vont à contre-courant de la dynamique locale qui se tourne, dans un élan de modernisme, vers le centre. De ce point de vue, la situation ne semble guère avoir évolué depuis la remarque de Marius Barbeau en 1945 :

Les contes, les chansons, les métiers de la terre, de la rivière et des bois, les coutumes d'autrefois, le langage ancestral, tout est jeté de côté sous la poussée du progrès. C'est justement ce que le progrès fait disparaître que le chercheur amasse, et dont il veut surprendre le souvenir, l'expression, la forme entière avant qu'ils meurent ${ }^{43}$.

Conflit constant entre la périphérie désireuse de rénover son image, justement pour sortir de sa marginalité, et le centre qui la reconnaît pour son passé...

\section{Causes et conditions de la résistance}

\section{Les causes}

Et si le principe du limaçon était avéré, à quelles causes attribuer la vitalité du patrimoine des migrants et des groupes marginaux ? À un stimulus déclenché par le choc migratoire ? Ou aux souvenirs indélébiles qui survivent en dépit de l'éloignement du pays natal et qui, pour cette raison, seraient plus fidèlement transmis ? Ou encore, tout simplement, au prolongement d'un climat favorable à leur épanouissement ? La persistance de certaines traditions en milieu minoritaire, dans les marges de la francophonie-1'Acadie par exemple ou l'Ontario français -, expose, il est vrai, la mémoire plus longue des migrants qui, passant d'un continent ou d'un pays à un autre, ou d'une province à une autre, n'ont pu souvent apporter avec eux que le bagage de leurs traditions orales. Le

\footnotetext{
1963, dont l'existence remonte à 1886 au Collège Memramcook) et, depuis peu, de la chaire de recherche McCain en ethnologie acadienne (2006). Mais aucun programme de premier ou de deuxième cycle n'unifie encore cet enseignement. Aussi, son premier doctorat, lancé en 1991, fut-il en études françaises (subdivisé en 2004 en études littéraires et en sciences du langage)... comme nous l'a précisé le directeur actuel du Département d'études françaises, Raoul Boudreau, l'Institut d'études acadiennes, créé en 2006, annonce la mise en place d'un programme de maîtrise en études acadiennes.
}

43. Marius Barbeau, « En quête de connaissances [...] », op. cit., p. 41. 
conservatisme de ces migrants, centrifugés en périphérie, s'inscrit dans la continuité par un attachement tout naturel à ce qu'ils sont et à ce qu'ils connaissent. En somme, s'ils conservent plus longtemps et mieux les traditions que les populations du centre qui les oublient ou les rejettent, c'est moins d'abord par une action volontaire que par une résistance inconsciente et, surtout, par la présence de conditions favorables.

\section{Les conditions}

Parmi les circonstances qui concourent à cette résistance des marges, la masse critique paraît fondamentale. Par exemple, une immigration massive, réunissant par un sort commun et en un même lieu des individus de même origine qui parlent une langue commune, partagent les mêmes croyances et exercent entre eux la solidarité par des activités d'entraide, formerait éventuellement une société cohérente par son unité ou peut-être son homogénéité culturelle. L'expérience collective qui en résulterait saurait reconnaître les éléments étrangers du groupe afin de les repousser au besoin ou, avec le temps, de les intégrer. L'établissement de très nombreux villages en Amérique française repose sur cette communauté d'origine: les Acadiens de Saint-Martinville en Louisiane, les Wallons de Brussels au Wisconsin, de SaintAlphonse et de Bruxelles au Manitoba, les Canadiens français et les Acadiens de Fort-Kent au Maine, les Canadiens français de Lafontaine en Ontario, les Franco-Américains de Plamondon en Alberta ou les Bretons de Saint-Brieux en Saskatchewan; des migrations comparables vers les villes ont aussi composé des quartiers français plus ou moins homogènes, tels le Moulin-àFleur à Sudbury et les Petits-Canadas en Nouvelle-Angleterre, ou favorisé des concentrations à proximité qui ont fini par s'intégrer à des centres : Dieppe à Moncton, Orléans à Ottawa, Saint-Boniface à Winnipeg, ou Maillardville à Vancouver.

La masse critique sera renforcée par l'isolement et la relative autonomie qui en découle. Les villages de pêcheurs, les agglomérations agricoles, minières ou forestières sauvegarderont, 
même dans la dépendance économique, la cohésion sociale que procure leur forme particulière d'insularité. Des facteurs comme la xénophobie, le racisme, l'ostracisme et les autres formes de ségrégation ou de rejet renforceront parfois la marginalisation ethnique, sociale ou religieuse : on l'a observé chez les « hérétiques » de Girardville ou les survivants du protestantisme francophone de la paroisse Pinguet de Saint-Damase-de-L'Islet au Québec ${ }^{44}$, comme chez les Métis de Saint-Laurent au Manitoba $^{45}$. Cette insularité, soutenant la reproduction du mode de vie traditionnel, se vit toutefois dans des conditions matérielles rudimentaires, voire dans la pauvreté, puisqu'on s'applique d'abord à transplanter tant bien que mal des habitudes prises ailleurs et nécessitant force adaptations au nouveau pays, ce qui emploie le plus clair des énergies disponibles. Primo vivere plutôt que thésauriser, voilà qui résume alors l'humble et essentielle activité du peuple.

Une telle situation garantit néanmoins une suffisante stabilité du groupe qui le garde durant une période plus ou moins longue, parfois des siècles, à l'écart - ou à l'abri - des changements qui surviennent dans le milieu d'origine : guerres et bouleversements sociaux, comme ceux engendrés par la Révolution française, conflits politiques, transformations sociales affectant le travail et la famille, évolution de la langue et des mentalités qui s'alignent sur les progrès techniques, les moyens de communication et les modes nouvelles.

L'inscription durable dans le temps intensifiera l'interdépendance des membres de ces collectivités insulaires et créera une appartenance véritable. Le rapprochement issu de la parenté et du voisinage, chevillé par l'histoire au territoire partagé, forme la trame d'une conscience communautaire, expression d'un

44. «Canadiens français quoique protestants », dans Jean Simard, Le Québec pour terrain. Itinéraire d'un missionnaire du patrimoine religieux, [Québec], PUL, « Archives de folklore » 28, 2004, p. 73-83; voir aussi l'historique de cette paroisse à l'adresse www.egliseunie.org/paroisses/pinguet/historique.htm, un document préparé par Geneviève Trudel, 2002.

45. Guy Lavallée, The Metis of St. Laurent, Manitoba : Their Life and Stories, 1920-1988, Winnipeg, The Author, 2003, XVII-171 p. 
« conservatisme inné ${ }^{46}$ », et de l'univers de références sociales qui s'en dégage. Il en résulte la fierté de son appartenance et le souci de la continuité. L'exemple contemporain de l'opposition des résidents de Saint-Joachim et de Pointe-aux-Roches, dans le Sud-Ouest ontarien, habitants pourtant paisibles et ordinairement respectueux de leur clergé et de l'autorité qu'ils lui reconnaissent, en fournit la parfaite illustration. Devant la menace de destruction qui planait sur leurs églises, un patrimoine doublement « sacré » à leurs yeux, ces paroissiens en sont venus, dans un réflexe d'autodéfense, à contester ouvertement les décisions du diocèse de London, par la voie des tribunaux. Considérant que leurs temples étaient des «ports » d'attache, des «ponts » entre les générations et des "phares» de leur identité française, ils organisèrent une résistance intelligente, appuyée sur une argumentation solide, concertée avec le milieu, guidée par des avocats chevronnés et médiatisée à la grandeur de l'Ontario, en dépit de l'absence de tout intérêt économique. Durant l'automne 2007, au terme de dix années de lutte, le collectif Sos-Églises obtenait finalement gain de cause ${ }^{47}$. Résistance peut-être « incompréhensible » pour plusieurs ${ }^{48}$, mais certainement née de la conscience de sa différence, et en vertu de sa fidélité à sa foi, à sa langue, à ses traditions et à sa patrie, telle que le proclamait naguère une Église disparue.

Ainsi, une tradition qui se transmettait par habitude, par goût, sans y penser presque, surgit à la conscience comme élément fondamental du noyau, ou parfois de son enveloppe, d'une différence dont l'Autre est le déclencheur. Pour la collectivité, la perception de son altérité se compare à celle de l'artiste en

46. Expression de Marius Barbeau, Romancero du Canada, op. cit., p. 156, à propos d'une chanson de fileuse : « Ce qui souvent s'est perdu là-bas s'est conservé ici, grâce à l'isolement et au conservatisme inné du colon canadien. »

47. Sur l'expérience de Sos-Églises, voir les réflexions d'André Chénier et de David Tremblay regroupées sous le titre « Quand l'Église démissionne », Le Patrimoine religieux de la Nouvelle-Écosse. Signes et paradoxes en Acadie. Actes du colloque national organisé les 19 et 20 juin 2006 à l'Université Sainte-Anne, sous la direction de J.-P. Pichette, Port Acadie. Revue interdisciplinaire en études acadiennes, $\mathrm{n}^{\mathrm{os}} 10$ 11-12, automne 2006-printemps 2007-automne 2007, p. 243-272.

48. Voir en annexe ce que constate Diéreville au sujet des Acadiens. 
présence de son public, ou du vacancier hors de son milieu de travail, qui choisit délibérément son exclusion de la vie normale et l'interprète comme un signe d'émancipation personnelle plutôt que de subordination. Quand, par aventure ou par dessein, surviendra un étranger, voyageur ou chercheur, dans ce terroir isolé, mais cohérent et stable, il jugera cette marge conservatrice, archaïque, « inchangée », « stationnaire », « figée », voire « momifiée », retardée peut-être ou en état d'hibernation, en tout cas offrant un aspect exotique dont le centre s'est délesté en chemin, et il admirera sa " résistance », sa « richesse », son « foisonnement », sa « fraîcheur ». Fasciné par la longévité remarquable de ses traditions, il en recommandera l'examen approfondi dans un « laboratoire » qu' on ne croyait plus possible ${ }^{49}$.

\section{L'érosion de la marge}

Les peuples émigrés n'ont bien évidemment pas conservé toutes les traditions du pays d'origine ; beaucoup s'en faut et, si celles que nous signalons dans cette étude ont résisté, d'autres, peutêtre plus nombreuses, n'ont vraisemblablement pas survécu. En ce sens, on peut dire qu'il y a effectivement eu déperdition, un phénomène naturel, propre à tout organisme vivant. Les remarques de Rémy de Gourmont à propos de la «Claire fontaine » et de « Vive la Canadienne », deux chansons qu'il jugeait inférieures à la tradition française, en sont les premiers signes. Marguerite et Raoul d'Harcourt, qui célébraient avec passion la mémoire exceptionnelle du répertoire lyrique du Canada français, n'en avaient pas moins souligné quelques carences : «On s’étonnera peut-être de certaines lacunes : nous ne donnons aucun chant de labour, aucune berceuse, aucun noël. Ici la carence est imputable au Canada. Les chants de labour y sont inconnus [...]. Les berceuses sont rares et les noëls peu nombreux ${ }^{50}$. " Récemment, après un séjour en Ontario, où elle entendit avec émotion la

49. La plupart de ces expressions sont tirées des auteurs cités en annexe.

50. Marguerite et Raoul d'Harcourt, Chansons folkloriques françaises au Canada. Leur langue musicale, [Préface par Marius Barbeau. Prélude aux chansons par Olivier Messiaen], Québec, Pul ; Paris, Puf, 1956, p. 55. 
première version vivante du «Flambeau d'amour», Bárbara Fernández formula le paradoxe qu'elle avait aussi rencontré dans la tradition orale de son pays : « la vitalité et la vigueur de la tradition orale iraient à l'opposé et au détriment de la qualité poétique de la chanson jusqu'à détruire la capacité d'évoquer des images fortement symboliques et d'aller même à changer la signification profonde du récit ${ }^{51} »$.

L'exemple du village acadien de Chezzetcook, qu'a étudié l'ethnologue Ronald Labelle, représente peut-être le plus justement ce paradoxe ${ }^{52}$. Tant que durèrent l'éloignement du centre, avec l'isolement relatif et l'autonomie qui en découlent, l'usage d'une langue particulière et d'une religion différente, en même temps que les traditions fortes que le couple langue et religion imprègne, une forme de rejet, voire de discrimination par la majorité, dont la négligence se traduit par l'absence de routes et de services publics, et les difficultés économiques qui s'ensuivent et qui sollicitent la débrouillardise collective, la cohésion sociale de cette petite communauté française de la Nouvelle-Écosse dura et les traditions résistèrent vraisemblablement là mieux qu'ailleurs. Le rapport de l'historien Rameau de Saint-Père, qui visita en 1860 ce village de « 1400 habitants français », fournit des données précises sur la plupart de ces facteurs : «ils ont parfaitement conservé notre langue, et leur costume est même plus voisin de celui de nos campagnards qu'aucun autre que j'aie rencontré chez les Canadiens ou les Acadiens. » Il remarque même que « leurs femmes, seules entre toutes les Acadiennes, portent une sorte de coiffure en mousseline blanche comme un voile drapé autour de la tête dont je ne connais l'analogue nulle part». Il s'étonne alors de leur « singulière conservation de la langue, du costume et des mœurs », qu'il juge

51. Bárbara Fernández Taviel de Andrade, « "Le Flambeau d'amour” au Canada : conservation et innovation ", Rabaska, revue d'ethnologie de l'Amérique française, vol. 2, 2004, p. 49.

52. Ronald Labelle, La Vie acadienne à Chezzetcook, Nouvelle-Écosse, Cahiers de la Société historique acadienne, vol. 22, $\mathrm{n}^{\text {os }}$ 2-3, avril-septembre 1991, 95 p. 
« d'autant plus surprenante qu'ils sont entièrement séparés de tous les autres établissements acadiens, très éloignés, et privés de toute communication avec aucun autre centre français ». Mais ils sont également isolés de la population anglophone locale : « Les côtes en effet sont aux environs d'Halifax très abruptes et d'une fertilité médiocre et assez peu peuplé[es], de telle façon que les gens de Chez[z]etcook sont à peu près sans voisins, et sans autres relations que les petits voyages qu'ils font à Halifax sur leurs barques pour les nécessités d'achat[s] et de ventes. » Il notera encore que, «[c]omme tous les Acadiens, ils sont très attachés à la religion catholique et à leur nationalité ». Selon lui, leur « isolement extrême » expliquerait leur « originalité de costume et de langage qui semble en faire un spécimen paléoontologique de la famille acadienne telle qu'elle pouvait être il y a un siècle ${ }^{53} »$.

Le « grand privilège de conserver plus intacts qu'aucun autre les caractères spéciaux de leur origine » aura fini par s'éroder avec le temps à telle enseigne que l'enquêteur des années 1980 écrira : "Aujourd'hui, le contraste est étonnant. Seules les anciennes maisons rappellent les origines acadiennes de ce village où, après deux siècles d'efforts, la population semble s'être résignée à voir sa culture disparaître ${ }^{54}$. » La pauvreté et la discrimination ont poussé plusieurs de ces Acadiens à émigrer vers les États-Unis puis vers d'autres villes canadiennes jusqu'à la Deuxième Guerre mondiale ; par la suite, l'amélioration du réseau routier et les rapports plus constants et utilitaires avec le milieu anglophone d'Halifax auront amené le bilinguisme, les mariages mixtes et, conséquemment, le « déclin rapide » de la langue et de la culture françaises chez cette minorité qui garde néanmoins encore « une identité propre». Aussi l'auteur ne peut-il que « partager la vision fataliste des anciens qui voient leur langue et leur culture disparaître ${ }^{55} »$.

53. Toutes ces citations sont extraites du livre de Ronald Labelle, La Vie acadienne à Chezzetcook, Nouvelle-Écosse, op. cit., p. 12-14.

54. Ibid., p. 15.

55. Ibid., p. 85-86. 


\section{Le complexe de la marge}

Chacun sait combien toute minorité éprouve de grandes difficultés à se faire connaître et surtout reconnaître par la majorité, car les marques de sa présence se propagent peu dans l'architecture, dans les arts, qu'elles sont absentes des canaux médiatiques et n'ont guère d'influence sur la vie nationale, la politique ou l'histoire. Entre deux mondes auxquels les marges participent - provenant de l'un, mais vivant dans l'autre ; tantôt de l'un, tantôt de l'autre ; n'appartenant totalement ni à l'un ni à l'autre; négligées par le centre de l'un et le centre de l'autre -, elles vivent en retrait, loin des prises de décision qui affectent la collectivité, en retard sur les mouvements économiques, sociaux, culturels. Cette mise à l'écart, qui est souvent cause d'impuissance, de frustration, de colère, attisera néanmoins la débrouillardise et inspirera la prise en main indépendante, source d'un dynamisme local propice à l'innovation et d'une identité régionale forte dont on tirera sa fierté. Mais son pendant négatif est le complexe d'infériorité. Pascal Poirier, dans la préface de l'étude qu'il consacra à la langue acadienne, a bien saisi cette altération qu'il applique au comportement linguistique.

Certaines expressions dont on se sert, en Acadie, font sourire les Canadiens et les « Français de France ». Nos gens n'aiment pas qu'on se moque d'eux. Plutôt que de s'exposer à la risée des étrangers, ils se tairont, ou parleront anglais ; ou bien, s'ils parlent français, leur français, ce sera avec gêne, presque en rougissant. / Et pourtant, cette langue, c'est celle que leurs pères ont apportée de la Touraine et du Berri, dans la première partie $\mathrm{du} \mathrm{XVII}^{\mathrm{e}}$ siècle, lorsqu'ils vinrent coloniser « la NouvelleFrance » d'Amérique. / La crainte qu'éprouve le paysan acadien de parler sa langue devant les étrangers[,] et même en présence de toute personne " éduquée », est chose dangereuse pour lui, au point de vue national. Elle ouvre une écluse à la marée montante de l'anglicisme, qui déjà déborde ${ }^{56}$.

56. Pascal Poirier, Le Parler franco-acadien et ses origines, [Québec, Imprimerie franciscaine missionnaire, 1928], p. 7. 
Il s'emploie alors à dépister certains particularismes contemporains dans les premiers monuments de la langue française. Si le parallèle est admissible entre les formes acadiennes et les documents originels sollicités - les adverbes icit (ici) et autre (autrement), le verbe et le nom aïde (aide), et le subjonctif faise (fasse) qu'il trouve dans le Serment de Strasbourg de 842, comme encore les tournures (par les petits, petit à petit), les prononciations (tchi, qui ; pleier, plier) et les mots (sus, sur ; adonner, convenir) que le IX ${ }^{\mathrm{e}}$ siècle lui fournit -, la continuité entre ces formes du moyen âge toujours en usage en Acadie serait plus difficilement démontrable ${ }^{57}$. Pourtant, le parler acadien, «la langue d'oui, le "français de Paris", allègue toujours Poirier, avait été transplanté sur les bords de la baie Sainte-Marie » plus d'un demi-siècle avant la parution de la première grammaire française et avait été, «à partir de 1710, complètement isolé du reste du monde, [...] n'étant venu en contact avec celui des Canadiens qu'après le pacte de $1867^{58} \gg$. Voilà comment cet auteur de la marge entend ranimer, par son « essai de réhabilitation du parler franco-acadien », la fierté collective en justifiant l'enracinement historique du français acadien, quitte à accepter sa conviction pour preuve.

Par le conservatisme, rendu notoire par les témoignages nombreux et tardifs relevés, qu'on voit singulièrement à l'œuvre chez les peuples déracinés, ceux qui sont les plus menacés dans leur existence par leur petitesse et leur différence, les marges affichent en certaines de ces matières culturelles une résistance inégalée. N'empêche qu'elles vivent vraiment, et de plus en plus, sur la corde raide, tant elles sont aussi fascinées par le centre et ouvertes à toutes les nouveautés. 


\section{II - Autres Temps, AUTRes MARGes ${ }^{59}$}

Parmi un étalage qui déploie de larges perspectives, quelques cas exemplaires que nous trouvons chez des minorités d'ici et d'ailleurs sauront exposer l'ampleur de cette résistance des marges. Ces applications supplémentaires du principe du limaçon débordent, pour la plupart, le strict domaine de l'oralité, puisqu'elles englobent le champ des pratiques coutumières et même celui de la culture matérielle.

\section{A. Amérique française}

De ce point de vue, bien des traditions de la diaspora française en Amérique révèleraient des facettes inédites, si elles étaient mieux étudiées par les chercheurs. Voyons-en quelques-unes.

\section{La " Guillonnée " au Missouri}

Dans l'introduction de ses contes populaires du Missouri, recueil capital publié en $1937^{60}$, Joseph-Médard Carrière écrivait que « la Guillonnée est la tradition la plus pittoresque qui ait survécu » dans cette région du Mississipi. Pratiquée par un groupe qui faisait la tournée des maisons la veille du jour de l'An et qui chantait «Bonjour le maître et la maîtresse » sur la musique d'un joueur de violon, cette quête se déroulait à plusieurs endroits : à Vincennes (Indiana), à Prairie-du-Rocher (Illinois) et à SainteGeneviève (Missouri). Toutefois, Carrière notait qu'elle perdait de plus en plus de terrain et qu'elle allait bientôt disparaître. Malgré ses sombres prédictions, fondées sur les terrains sérieux qu'il y avait faits de 1934 à 1936, cette tradition s'est maintenue jusqu'à nos jours comme l'attestent l'existence des « Sainte-

59. Ce thème a été au cœur des premières journées d'études de la COFRAM, tenues à l'Université Sainte-Anne (Pointe-de-l'Église, 25-26 octobre 2005), puis des Rencontres britto-franco-canadiennes qui ont suivi à 1'Université de Bretagne occidentale, « Traditions, revitalisations, créations » (Brest, 5-6 juin 2006) et enfin du colloque international « La Résistance des marges » (Pointe-de-l'Église, 15-20 août 2007).

60. Joseph-Médard Carrière, Tales from the French Folk-Lore of Missouri, Evanston and Chicago, Northwestern University, « Northwestern University Studies in the Humanities » 1, 1937, p. 6-7 [traduction de l'auteur]. 
Geneviève Guignolée Singers ${ }^{61}$ » et les recherches récentes effectuées dans ces mêmes lieux par Anna Burns pour la thèse qu'elle vient de soutenir ${ }^{62}$. Malgré le recul de la langue et les interdictions municipales qui ont voulu l'abolir, à chaque veille du jour de l'An, les descendants des pionniers français de SainteGeneviève, dans des costumes "bizarres et archaïques ", continuent d'aller de maison en maison et de bar en bar chantant toujours la même chanson ${ }^{63}$; ils y sont les bienvenus et boivent jusqu'au petit jour alors qu'ils rentrent à la maison prendre un peu de repos avant la messe du matin. Ainsi, survit à l'écart une guignolée qui serait disparue comme telle en France et qui serait généralement en régression au Canada français ${ }^{64}$. L'appui des groupes caritatifs au Québec a toutefois renouvelé cette tradition en une forme plus actuelle ramenée au début de décembre avec le concours de personnalités publiques : la Grande Guignolée des médias $^{65}$.

\section{La ceinture fléchée dans l'Ouest}

S'il est un symbole identitaire fort qui a grandement impressionné les étrangers dans les premières décennies du régime anglais, c'est bien « la ceinture de l'habitant du Bas-Canada, qui était partie intégrante de son costume traditionnel » et qui est « passée chez

61. Voir le site www.pbs.org/riverofsong/artists/e2-ste.html, toujours actif le 12 octobre 2009.

62. Anna L. Burns, "Bonsoir le maître et la maîtresse " : le rôle de la Guiannée dans le maintien des communautés franco-américaines de Sainte-Geneviève et de la Prairie-du-Rocher, Thèse (Ph. D.), University of Louisiana at Lafayette, 2009, 356 p.

63. Dans un article portant sur ce thème, Fañch Postic écrit que la tradition se poursuit ininterrompue à Prairie-du-Rocher, en Illinois : « Les quêteurs d'un jour, qui ne parlent plus le français, se font un devoir d'apprendre phonétiquement la chanson » ; $c f$. «Des marges atlantiques d'Europe aux francophonies d'Amérique du Nord. Les avatars d'une quête chantée : de "l'eginane" à la "guignolée" ", dans La Résistance des marges, op. cit., p. 444-445.

64. Marcel Bénéteau et Peter W. Halford, Mots choisis, op. cit., p. 276-277 : à l'article « Guignolée », du lexique du Détroit, on peut lire : « La pratique s'effectue aujourd'hui, là où elle vit toujours, en voiture, bien que le terme ne soit plus utilisé. »

65. Fañch Postic, "Des marges atlantiques d'Europe aux francophonies d'Amérique du Nord », op. cit., p. 443. 
les Amérindiens par le biais de la traite des fourrures ${ }^{66} »$. Ce transfert s'avère aujourd'hui heureux pour la continuité de la coutume, car, comme le signale Monique Genest-Leblanc, qui en a suivi la carrière sur " plus de deux siècles d'existence, la ceinture fléchée, au Québec et dans l'ouest du pays, occupe peu d'espace dans la mémoire collective du peuple qui en fut la source $^{67} \gg$. Elle se réduit en effet à la ceinture du Bonhomme Carnaval à Québec, comme dans d'autres festivals, à quelques pièces dans les collections muséales ou à de rares insignes honorifiques ; oubliée au grenier depuis la Révolution tranquille, en même temps que les stéréotypes de servitude, d'ignorance ou de "quétainerie » passéiste, elle est totalement proscrite du discours public, sinon pour condamner un esprit jugé trop étroit ou "pure laine ", de sorte que bien peu de Québécois et de Canadiens français trouveraient de bon ton de s'en parer au cours d'un événement officiel ${ }^{68}$. C'est tout le contraire chez les Amérindiens, qui la considèrent avec respect, et, surtout, chez les Métis, qui ont fondé l'Ordre de la ceinture fléchée. Le lieutenantgouverneur du Manitoba nommé en 1993, le Métis Yvon Dumont, se faisait un point d'honneur de la porter fièrement comme vêtement d'apparat, à l'image de Louis Riel et en souvenir des «voyageurs » français, leurs ancêtres, comme le font aussi les autres Métis, jeunes et vieux, dans les occasions spéciales ${ }^{69}$. Ce signe identitaire des Métis de l'Ouest canadien a fait le chemin à rebours puisque, adopté comme élément du costume officiel de

66. Monique Genest-Leblanc, "Une jolie cinture à flesche ». Sa présence au Bas-Canada, son cheminement vers l'Ouest, son introduction chez les Amérindiens, [Québec], Pul, «Ethnologie de l'Amérique française », 2003, p. 7 et aussi p. 82.

67. Ibid., p. 167.

68. Luc Lacourcière avait choisi une ceinture fléchée stylisée, dont la couleur varie selon les siècles, pour encadrer la couverture de la collection du « Nénuphar» (aux éditions Fides de Montréal) vouée aux «meilleurs auteurs canadiens », c'est-àdire aux classiques de la littérature canadienne-française, qu'on y réédite depuis 1944. Pour sa part, l'ethnologue et collectionneur Robert-Lionel Séguin (1920-1983) aimait porter le fléché traditionnel comme on peut le voir par les poses publiées dans les mélanges posthumes qu'on lui a offerts : La Vie quotidienne au Québec. Histoire, métiers, techniques et traditions, sous la direction de René Bouchard, Sillery, Presses de l'Université du Québec, 1983, p. [50] en patriote, et p. 60 en interprétant une danse traditionnelle.

69. Guy Lavallée, The Metis of St. Laurent, Manitoba, op. cit., p. 156-157.

volume 82010 
toute association métisse, il a fini par rallier les Métis des Maritimes, qui l'arborent désormais avec fierté ${ }^{70}$.

\section{La mi-carême en Acadie}

Disparue en France au tournant du $\mathrm{Xx}^{\mathrm{e}}$ siècle, la mi-carême a résisté chez les Français d'Amérique jusqu'à la Deuxième Guerre mondiale, après quoi elle s'est graduellement éteinte dans les régions centrales au profit de modes popularisées par le commerce ; on assiste depuis les années 1950 à la montée dans les centres urbains d'une mascarade d'automne, l'Halloween, avec son battage médiatique et sa panoplie d'objets de pacotille. Au Québec, la tradition ancienne n'a réussi à perdurer que dans trois endroits isolés, l'Isle-aux-Grues, qui fait face à Montmagny, sur la rive sud en aval de Québec, et deux régions du golfe du SaintLaurent reliées à l'Acadie par leur peuplement : Natashquan et Pointe-Parent sur la Côte-Nord, et Fatima aux Îles-de-laMadeleine ${ }^{71}$. Mais elle est encore très vivante dans quelques villages de la Nouvelle-Écosse et, pour l'une de ses formes, à Tignish dans l'Île-du-Prince-Édouard, là encore dans des zones côtières marginales, d'ailleurs très éloignées les unes des autres. Chez les Acadiens, elle a survécu aux interdictions de l'Église, à la disparition de l'observance stricte du carême et aux modes commerciales de l'Halloween. Georges Arsenault en a décrit les quatre formes qu'il a rencontrées : la distributrice de friandises en solitaire, la croque-mitaine, la cigogne qui apporte les bébés et la mascarade de la mi-carême, qui se court en groupe de maison en maison ${ }^{72}$. Cette dernière a si bien résisté qu'à Saint-Josephdu-Moine et à Chéticamp (Île-du-Cap-Breton), en NouvelleÉcosse, elle a progressivement occupé de plus en plus d'espace,

70. «Célébration de la journée des autochtones », Le Courrier de la NouvelleÉcosse, Pointe-de-l'Église, ${ }^{\text {er }}$ juillet 2005, p. 1, et « Journée autochtone célébrée en grand par des Métis acadiens », ibid., p. 10. Les Métis photographiés (p. 1 et 10) portent la ceinture fléchée à la taille tandis que les femmes la portent en bandoulière.

71. Pierre Dunnigan et Francine Saint-Laurent, Mi-Carême. Une fête québécoise à redécouvrir, [Montréal], Les 400 coups, [2006], 117 p.

72. Georges Arsenault, La Mi-Carême en Acadie, [Tracadie-Sheila, NouveauBrunswick], Éditions La Grande Marée, [2007], 161 p. 
passant d'un seul jour à toute une semaine, ne laissant à l'Halloween aucune chance de s'implanter solidement, et que les « maisons de mi-carêmes » accueillent plusieurs centaines, les plus populaires environ un millier, de visiteurs masqués. Ces dernières années, la création du Centre de la Mi-Carême a mobilisé les forces vives de cette communauté dans un projet de réveil économique pour neutraliser une crise importante du secteur de la pêche.

\section{Les petits pains de sainte Geneviève à Québec}

En plein cœur de la ville de Québec, une humble coutume religieuse continue à s'exercer, depuis des siècles, croit-on, à l'église Notre-Dame-des-Victoires. Il s'agit de la bénédiction des " petits pains de sainte Geneviève », le 3 janvier, jour de la fête de la sainte patronne de Paris, qui attire dans la petite église de la place Royale une foule nombreuse venue faire sa provision pour l'année qui commence. « Grâce à son intercession, Québec fut préservée de la famine comme l'avait été la ville de Paris au $\mathrm{V}^{\mathrm{e}}$ siècle », peut-on lire dans le dépliant destiné aux visiteurs. Ces " petits pains », gros comme le bout du pouce, que les croyants emportent en petite quantité et distribuent à leurs parents et à leurs connaissances, ont la propriété de protéger du manque de nourriture et, partant, du travail ou de l'argent qui la procure, ceux qui les portent sur soi dans leur bourse ou étui à chapelet. Ce rappel contemporain de la distribution gratuite de pains aux pauvres un millénaire et demi plus tôt par la vierge de Nanterre ne subsisterait que de ce côté-ci de l'Atlantique et vraisemblablement dans cette seule paroisse ${ }^{73}$. Or, nous avons découvert deux témoignages qui établissent bien 1'origine française de cette dévotion. En visite à Paris le 28 juin 1747, la Dauphine, après avoir entendu la messe à Notre-Dame, se rend à l'église SainteGeneviève où « on a découvert la châsse de sainte Geneviève en

73. Bernadette Guichard, rédactrice du tome I, vol. 8 du Manuel de folklore français contemporain d'Arnold Van Gennep (Paris, Picard, « Grands Manuels Picard », 1988), rencontrée en mars 2003, confirmait n'avoir jamais décelé cette tradition dans la documentation du fonds Van Gennep. 
entier » exprès pour elle. Après qu'on eut entonné les hymnes pour cette circonstance exceptionnelle, l'abbé présenta alors « un bassin d'argent où il y avoit deux boîtes pleines de petits pains de sainte Geneviève » à la visiteuse royale qui « en a pris un et l'a mangé dans 1 'église ${ }^{74} »$. Telle est la première mention. La deuxième est tirée du mémoire qu'Henri Grégoire, évêque de Blois, a préparé sur la «Révolte du clergé dissident contre le Concordat », dans lequel il critique certains des abus dont le clergé s'était rendu coupable ; il déplore notamment, dans le diocèse de Paris, que « [d]es momeries, ou tout au moins des dévotionnettes, remplacent la piété. Au lieu de se borner à prier, à imiter sainte Geneviève, [...] [q]uelle nécessité de vendre encore des petits pains de sainte Geneviève, de tromper le public, et de faire calomnier la religion par des prétendues reliques de sainte Geneviève $^{75}$ ? » Point question de bénédiction solennelle ni de distribution gratuite dans ces contextes ; mais, deux cents ans plus tard, l'expression « petits pains de sainte Geneviève », qui montre bien que le sacramental est le même, ne survivrait-elle qu'à Québec?

\section{B. Amérique anglaise}

Que ces populations de l'Amérique française conservent dans les marges du Missouri, de l'Ouest canadien, de l'Acadie et du Québec ces anciennes pratiques culturelles centrifugées de France puis du cœur du Québec - du moins pour la guignolée, la micarême et la dévotion aux petits pains de sainte Geneviève ${ }^{76}-$,

74. Mémoires du duc de Luynes sur la cour de Louis XV (1735-1758) publiés sous le patronage de M. le duc de Luynes par MM. L. Dussieux et E. Soulie, Paris, Firmin Didot frères, fils et Cie, 1861, tome vIII, 1747-1748, p. 254.

75. Mémoires ecclésiastiques, politiques et littéraires de M. Grégoire, ancien évêque de Blois (rédigés en 1808) [député à l'Assemblée constituante et à la Convention nationale, sénateur, membre de l'Institut], Paris, J. Yonet libraire, 1840, tome II, p. 424.

76. Pour le port de la ceinture fléchée, les travaux de Monique Genest-Leblanc, basés sur la technique particulière du tissage aux doigts, montrent qu'elle serait née dans le Bas-Canada au XVIII ${ }^{\mathrm{e}}$ siècle, non en France ou en Europe où existaient cependant des ceintures fabriquées selon d'autres techniques. Voir la conclusion de son livre «Une jolie cinture à flesche », op. cit., p. [165]-167. 
est indubitable et vérifiable par les sources avancées. Les marges résistent donc à peu près partout, comme la danse sur les bas et la danse dans l'auge en Ontario français. Sauf que la résistance n'est pas 1'apanage exclusif des Français d'Amérique ${ }^{77}$. Ces dynamismes, illustrés à partir de traits particuliers à la francophonie, agissent aussi au sein d'autres minorités périphériques, chez d'autres peuples émigrés, tels les Irlandais, les Écossais et les Anglais en Amérique du Nord. Loin de se limiter à un peuple donné, le principe du limaçon aurait ainsi une portée générale. C'est du moins ce que valident les quelques cas qui nous sont connus.

\section{La célébration de la Saint-Patrice}

Selon Nancy Schmitz, « la Saint-Patrice n'a pas toujours été une fête particulièrement importante pour les Irlandais vivant dans leur pays d'origine ${ }^{78} \gg$. Cette fête religieuse était même moins importante que des fêtes traditionnelles comme la Saint-JeanBaptiste, Noël et la Toussaint. Et ce n'est qu'avec l'immigration irlandaise en Amérique du Nord qu'elle prit un nouvel essor dans un contexte où il importait « de s'affirmer avec fierté face aux autres groupes en présence, dans un nouveau pays où toutes sortes de traditions ethniques se côtoient ». C'est là qu'apparut le défilé, « un élément tellement important dans plusieurs villes, telles que Montréal, New-York et Boston, qu'il a influencé le vieux pays

77. S'il fallait inclure les francophonies des Antilles, on trouverait d'autres cas de résistance. Par exemple, selon une thèse inscrite en ethnomusicologie à l'Université de Montréal par Marie-Hélène Pichette, la pratique musicale appelée gwoka en Guadeloupe serait la résultante des rythmes africains de tambour, avec les chants et les danses qu'il accompagnait, que les esclaves auraient maintenus dans des conditions impossibles, malgré la désapprobation générale, notamment cléricale, et adaptés aux instruments qu'ils pouvaient se fabriquer à partir de matériaux de récupération; cette tradition rallie aujourd'hui leurs descendants dans un festival très fortement identitaire.

78. Nancy Schmitz, «La Saint-Patrice : fête d'identité et de cohésion ethnique », dans Que la fête commence! Actes du colloque national sur la fête populaire organisé par la Société des festivals populaires du Québec 1980, Textes recueillis et publiés sous la direction de Diane Pinard, [Montréal], La Société des festivals populaires du Québec, [1982], p. 78. L'auteur reprendra cet article [p. 78-83] dans son livre Irish for a Day. Saint Patrick's Day Celebrations in Québec City 1765-1990, [Sainte-Foy], Carraig Books, [1991], XII-295 p. ; les extraits cités ici figurent aux p. 4, 7-9.

volume 82010 
lui-même », au point que la tradition du Nouveau Monde a été exportée en Irlande : «Il existe aujourd'hui à Dublin un défilé de la Saint-Patrice, emprunt nord-américain ${ }^{79} »$, assure l'ethnologue. C'est à se demander si la redécouverte de la tradition celtique de l'Halloween à partir des États-Unis et son expansion actuelle dans toute l'Europe, par une publicité hors du commun soutenue par des intérêts mercantiles évidents, ne relèverait pas d'une semblable dynamique.

\section{La gigue néo-écossaise}

L'abbé Eugene Morris, curé de la paroisse Sainte-Thérèse de Sydney au Cap-Breton, a été invité « à donner des cours intensifs en Écosse afin de faire revivre des danses de pas, originaires de cette partie de l'Écosse, encore vivantes en Nouvelle-Écosse, mais disparues de la mère patrie ». Il s'agissait bien de réintroduire ces danses au pays, afin de les présenter au festival de danse et de musique traditionnelles qui a lieu à Barra dans les Hébrides. En 1983, ce fut le maître à danser Mary Janet MacDonald, de Mabou, Nouvelle-Écosse, qui prit la relève et donna cette formation ${ }^{80}$. La réinsertion de gigues écossaises, maintenues par des émigrés dans la marge néo-écossaise alors que la «mère patrie » s'en était éloignée, manifeste une fois de plus l'érosion culturelle du centre et le conservatisme de la périphérie.

\section{Les traditions terre-neuviennes}

À plusieurs signes, dont la présence d'un département de Folklore à l'Université Memorial n'est certes pas le moindre puisqu'il en encourage le dépistage, Terre-Neuve se révèle une province très conservatrice de ses traditions. Le Mummering, une coutume des pays nordiques qui amalgame en quelque sorte la mascarade de la mi-carême et la quête de la guignolée, ne paraît tenir que dans cette partie du Canada anglais. Il en serait ainsi des feux du 5 novembre, appelés Bonfire Night, qui commémorent la tentative

79. Nancy Schmitz, « La Saint-Patrice [...]», op. cit., p. 79-80.

80. Simonne Voyer, La Gigue. Danse de pas, [Sainte-Foy], Les Éditions GID, [2003], p. 38. 
avortée du catholique Guy Fawkes d'incendier le palais de Westminster et de supprimer le roi protestant Jacques $\mathrm{I}^{\text {er }}$ en novembre 1605. Depuis, on célèbre cet échec chez les protestants - et même chez les catholiques - davantage à Terre-Neuve qu'en Angleterre, dit-on, en allumant des feux de joie un peu partout, voire dans les ordures ${ }^{81}$. Enfin, certains citoyens de cette province auraient tellement bien préservé l'accent de leur terre de départ qu'ils passent fréquemment au Canada pour des Irlandais plutôt que pour des Terre-Neuviens. L'un d'eux aurait vécu semblable expérience en Irlande même, alors que, dans un bar, on refusa de lui servir à boire sans avoir au préalable montré son passeport, persuadé que ce client mentait en se prétendant Canadien. Le garçon, qui avait reconnu l'accent typique d'une région de l'Irlande, était convaincu d'être en présence d'un espion de l'Armée républicaine irlandaise. La présentation du passeport permit de rétablir les faits ${ }^{82}$.

\section{Les chansons des Appalaches}

Entre 1916 et 1918, Cecil Sharp (1859-1924), considéré comme le père de la renaissance du folklore anglais, recueillait plus de 500 chansons, soit 1612 versions musicales, aux États-Unis, dans la partie sud des Appalaches : en Caroline du Nord, au Kentucky, en Virginie, en Virginie-Occidentale et au Tennessee. Avec le recul, cette exploration est particulièrement intéressante pour notre propos, tant on y reconnaît une saisissante application du principe du limaçon. D'abord, parce que Sharp avait prévu que ces régions, peu accessibles par des voies carrossables pendant plus d'un siècle, seraient un terreau fertile pour ses recherches, spécialement

81. Dans une entrevue à la radio de la SRC-Halifax, le 5 novembre 2007, Ronald Labelle, titulaire de la chaire McCain en ethnologie acadienne, à l'Université de Moncton, notait que la tradition est ambiguë, car les catholiques la célèbrent également, ce qui signifierait qu'on incendie le Parlement chaque année et que Guy Fawkes a finalement réussi son coup.

82. Renseignements confiés par Sean Collier, Terre-Neuvien étudiant à l'Université Sainte-Anne, et repris dans des courriels du 15 décembre 2005, puis du 23 janvier 2006. Récit d'expériences vécues dans sa famille maternelle, arrivée au Canada vers 1830 en provenance de Calvert, Irlande du Nord. 
là où des conditions rudimentaires subsistaient. Ce qu'il vérifia bientôt par la langue qu'on y parlait, un anglais à l'ancienne plutôt que l'anglo-américain ${ }^{83}$; ensuite, parce que les chansons étaient le lot des informateurs de tous les âges, des plus jeunes aux plus vieux, à la différence de ses collectes en Angleterre, où personne de moins de 70 ans ne connaissait le répertoire. « En fait, notera$\mathrm{t}-\mathrm{il}$, je me trouvai pour la première fois de ma vie dans un milieu où le chant était aussi commun et presque aussi universel que la parole $^{84}$. " Sa collaboratrice, Maud Karpeles, qui publia une compilation posthume de sa collecte, rapporte que Sharp avait aussi prévu que ces conditions favorables ne sauraient durer. De retour sur place en 1951, elle découvrit « avec quelle rapidité la culture spontanée des gens semble disparaître une fois qu'ils ont été en contact avec la civilisation moderne, et comme ils ont tôt fait d'imiter les manières et de s'imprégner des goûts du beau monde ${ }^{85} \gg$. Les routes, l'électricité, la radio avaient rattaché ces populations aux centres régionaux et répandu la musique à la mode, vouant à l'oubli l'ancien répertoire que les gens prudes dédaignaient carrément : « Nous nous sommes fait une règle de ne jamais jurer et de ne jamais chanter des chansons d'amour devant les enfants. » Pourtant, elle réalisa à quel point la tradition a la vie dure et qu'il suffit de peu pour en ranimer le souvenir : l'imprimé allait relayer la mémoire et faire rejaillir aux lèvres de l'interprète la pièce confiée au collecteur des décennies auparavant. Et Karpeles de conclure en exposant le cycle : «Thus, a song, originating in England and carried to America, lives there by oral tradition for some hundreds of years; it is written down and taken back to England by Cecil Sharp; then some thirty years later the song is carried back in printed form to the country of its

83. [Cecil Sharp et Maud Karpeles], English Folk Songs from the Southern Appalachians, Collected by Cecil J. Sharp, Comprising two hundred and seventy-four Songs and Ballads with nine hundred and sixty-eight Tunes Including thirty-nine Tunes contributed by Olive Dame Campbell, Edited by Maud Karpeles, London, Oxford University Press, [1932, 1966], p. XXII.

84. Ibid., p. XXv [traduction de l'auteur].

85. Ibid., p. XVI [traduction de l'auteur]. 
adoption and takes on a new lease of life. Such are the devious ways of tradition ${ }^{86}$. » Tels sont, en effet, les détours de la tradition.

À leur façon, ces cas reprennent en partie les conclusions de Peter Ward, qui avait aussi noté, dans son étude des rituels du mariage, leur persistance plus grande dans l'intimité des petites villes et des régions rurales du Canada anglais, dans les zones périphériques de l'Est ontarien et dans les Maritimes, parce qu'elles sont petites et isolées, à la différence de la grande mobilité et de l'anonymat qui caractérisent les centres populeux ${ }^{87}$.

\section{Conclusion}

Le principe du limaçon est-il l'exception qui confirme la règle ou bien la règle qui souffre des exceptions? À en juger par la danse de l'aîné célibataire, dont le cheminement séculaire et la vitalité nord-ontarienne ont donné naissance à cette réflexion, il y a tout lieu de convenir que la résistance des marges se situe du côté de la règle. Toutefois, nous l'admettons volontiers, une lecture critique de cet essai fera certes surgir des exceptions qui paraitront prendre le contre-pied de ce modèle, tellement il semble évident qu'on pourra documenter des traditions vivantes au sein des majorités comme, à l'inverse, repérer des traditions oubliées au sein des minorités. Le principe du limaçon n'est donc pas infaillible et ce n'était pas notre intention de le présenter comme tel. L'urbanisation massive et la disponibilité des communications ont érodé les clivages et produit une grande uniformisation qui met en danger les particularités régionales. Comme la maladie compromet la santé et la mort détruit la vie, l'érosion agit sans cesse et partout ; mais ces dégradations temporaires qui gênent une existence et ces déchéances définitives qui la perdent n'entravent guère la course de l'espèce qui se maintient et s'adapte : on ne prévoit pas encore que les générations à venir

86. Ibid., p. Xx.

87. Peter Ward, Courtship, Love and Marriage in Nineteenth-Century English Canada, Montréal \& Kingston, London, Buffalo, McGill-Queen's University Press, [1990], p. 118-119. 
seront moins humaines que les générations passées. Sans nier donc les possibilités d'érosion culturelle, le principe du limaçon met plutôt de l'avant les cas de résistance, moins apparents, et peut tout à fait s'interpréter comme une apologie de la marge.

Évidemment les derniers exemples rapportés ci-devant, tout autant que la pratique qui a déclenché notre enquête, n'adhèrent d'aucune façon à l'hypothèse de la simplification des rituels, telle qu'on l'a naguère avancée. Au contraire, l'analyse confirme que le rituel de la danse de l'aîné célibataire, tout particulièrement dans ses variétés franco-ontariennes, a plutôt débouché sur un foisonnement inattendu. Cette abondance transparaît d'abord par le maintien simultané ou successif de la plupart des formes attestées anciennement : la danse dans une auge, dans une cuve ${ }^{88}$ ou sur ses bas. Mais, surtout, elle s'accomplit par le déploiement d'une série d'innovations qu'un niveau de vie supérieur autorise de nos jours : les chaussettes tricotées spécialement pour la circonstance, l'ajout d'éléments ridicules pour les décorer et parfois d'autres ornements vestimentaires, l'enfilade rituelle des bas, l'allongement de la danse en solitaire avec, au besoin, le réconfort de membres de la famille, le jet de monnaie et son ramassage, la photographie et l'enregistrement audiovisuel de la scène, etc. Loin de l'inexorable appauvrissement d'un vieux rituel, qui aurait perdu son sens et se serait érodé au cours du peuplement des régions de l'Ontario, on observe plutôt, dans les territoires neufs de cette marge, la perpétuation et l'acclimatation d'un rite de passage ancien et, contre toute attente, une ritualisation amplifiée qui a fortement contribué à enrichir et à régénérer une pratique qui nous paraît avoir été beaucoup plus sobre autrefois.

88. Alors que l'auge est une réalité disparue des milieux urbains qui pratiquent aujourd'hui ce rituel, son maintien a de quoi surprendre, tant parce qu'on a été jusqu'à en fabriquer une exprès pour l'occasion que parce qu'on l'a remplacée par une cuve, un banc renversé, une boîte de carton ou tout récipient qui peut s'y prêter, voire un enclos, mais en conservant toujours la même expression de la « danse dans l'auge ». 


\section{AnNeXe : Regards et disCours SUR LA MARGE}

\section{A. Des « Français intacts »}

La résistance des marges ne serait pas une proposition nouvelle. De fait, les témoignages, en assez grand nombre, qu'ont consignés des observateurs étrangers auraient dû aiguiller les chercheurs vers l'examen de la thèse du conservatisme des Canadiens français, tant ils ont appuyé sur ce point. L'assurance de Gustave de Beaumont, de passage dans la région ontarienne de SaultSainte-Marie, en août 1831, fournit l'illustration typique de cette perception et, à ce titre, devrait porter à la réflexion.

Le peu de temps que j'ai passé avec les Canadiens m'a prouvé combien le caractère national, et surtout le caractère français, se perd difficilement ; la gaieté française qu'ils ont conservée tout entière contraste singulièrement avec le sang-froid glacial des Américains. Il est aussi à remarquer que les Français du Canada sont plus gais que nous ne le sommes maintenant en France ; la raison en est simple : leur situation a moins changé que la nôtre ; ils n'ont point passé à travers notre Révolution qui a tant influé sur la nouvelle direction que notre caractère national a pris[e]; ils n'ont point comme nous leur attention fixée sur des intérêts politiques qui les préoccupent exclusivement. Il est donc vrai de dire que, quant au caractère antique de la nation, ils sont plus Français que nous ne le sommes ${ }^{89}$.

Bien d'autres visiteurs ont exprimé, sur la persistance du caractère français au sein des populations canadiennes, pareille opinion dont on trouve une remarquable continuité durant plus d'un siècle et demi. Au milieu du XIX ${ }^{\mathrm{e}}$ siècle, un officier de la marine française, L. du Hailly ${ }^{90}$, découvrait un village acadien de la Nouvelle-

89. G. de Beaumont, Lettres d'Amérique 1831-1832, Paris, Puf, 1973, p. 122123. Cette source nous fut aimablement communiquée par Yves Lefier ${ }^{\dagger}$, professeur de littérature à l'Université Laurentienne.

90. L. du Hailly [pseudonyme d'Édouard-Polydore Vanéechout], Campagnes et stations sur les côtes de l'Amérique du Nord, Paris, E. Dentu, 1864, chapitre vi « Les Acadiens et la Nouvelle-Écosse », p. 183-184. Les extraits cités ici ont été repris par Faucher de Saint-Maurice, De tribord à babord. Trois croisières dans le golfe SaintLaurent. Nord et Sud, Montréal, Duvernay frères \& Dansereau, éditeurs, 1877, p. 233 236. Cf. Éd. Du Hailly, « Une station sur les côtes d'Amérique - III. Les Acadiens et la Nouvelle-Écosse », Revue des deux mondes, seconde période, Paris, tome 42, 1862 , p. 875-900, notamment p. 877-879. 
Écosse, situé à une trentaine de kilomètres à l'est de la capitale, Halifax. Constatant que «tout était français, tout avait été religieusement conservé, le costume aussi bien que le langage » à Chezzetcook, il se crut « transporté dans un village normand d'il y a deux siècles ${ }^{91} »$. La résistance de cette population laissée à elle-même l'amena à la réflexion suivante :

Serait-il vrai que l'attachement au sol natal se conserve d'autant plus vivace que la position sociale est moins élevée? Au lieu des humbles paysans dont nous parlons, supposons quelques opulentes familles françaises ayant échappé par hasard à la dispersion de leur race, et ayant depuis lors continué à s'enrichir : croit-on qu'elles ne seraient pas devenues aujourd'hui anglaises de mœurs, d'idées et de langage ${ }^{92}$ ?

On trouve une observation de même nature chez Rémy de Gourmont en 1889 dans son livre Les Canadiens de France :

Le naturaliste qui retrouverait en deux pays séparés par la mer la même flore et la même faune terrestre en conclurait nécessairement qu'en des temps plus ou moins éloignés, ces deux pays ont dû n'en former qu'un seul. La même conclusion ressortirait de ce seul fait que les mêmes chansons populaires se chantent au Canada et dans le nord-ouest de la France : les deux peuples jadis ont été un seul peuple. Faute d'autres documents, un point historique pourrait ainsi s'éclaircir, du moins d'une certaine lumière. Cela peut encore servir à prouver combien la tradition française est demeurée vivace au Canada, car c'est à peine, si, d'un continent à l'autre, apparaissent dans tel couplet de légères variantes ${ }^{93}$.

Ce personnage sera néanmoins l'un des rares à juger plus faibles certaines versions de chansons canadiennes : trouvant « moins saisissant » qu'un jeune homme soit abandonné et non une jeune fille, il écrira que « la Claire fontaine [...] qui se chante au Canada semble moins jolie que la version normande ${ }^{94} \gg$; et, à propos de

91. Ibid., p. 183.

92. Ibid., p. 184.

93. Rémy de Gourmont, Les Canadiens de France, Paris, Librairie de FirminDidot et cie, 1889, p. 213-214.

94. Ibid., p. 215. 
«Vive la Canadienne », il remarquera « que la chanson faite au Canada est assez inférieure à son modèle, hormis dans le premier couplet, dont on ne saurait dire de mal $^{95} \gg$. Il admettra certes que «[1] es traditions sont très tenaces chez le paysan canadien : il sait les chansons, il sait aussi les contes de la vieille France; il garde ses mœurs avec [...] jalousie ${ }^{96} \gg$. Il est d'ailleurs l'un des premiers Européens à se soucier des récits traditionnels : «Le conte fleurit au Canada, le vieux conte qui, depuis des siècles, amuse ceux qui n'ont que cette littérature et qui n'ont pas la plus mauvaise. » Conscient du peu de références disponibles, il prend soin d'ajouter : « Ce côté des mœurs canadiennes est assez peu connu : on a négligé de recueillir les contes comme on a recueilli les chansons. Néanmoins, nous en connaissons quelques-uns, grâce à $\mathrm{M}$. Carnoy à qui nul conte n'échappe ${ }^{97}$. " Il avait même proposé une explication à la popularité des traditions orales : « Pays très catholique, il [le Canada] ne pouvait pas toujours sympathiser avec notre littérature moderne ; dans ces conditions, le conte et surtout la chanson populaire devaient avoir beaucoup d'amateurs ${ }^{98}$. "

En 1910, la Revue franco-américaine publie, en réponse à une enquête de J. A. Lefebvre auprès de personnages français éminents, une série de témoignages qui révèlent « comment on nous voit en France ${ }^{99} \gg$. Ceux qui ont accepté de les rendre livrent inévitablement des avis favorables qui font des Canadiens français des exemples d'une fidélité digne d'admiration. C'est un Henry Simond, de l'Écho de Paris, qui déclare :

95. Ibid., p. 217.

96. Ibid., p. 231.

97. Ibid., p. 237-238; suit, au chapitre XVII, le conte « Le Cheval enchanté », p. 239-247.

98. Ibid., p. 217-218.

99. Après l'annonce, "Comment on nous voit en France ", Revue francoaméricaine, vol. 4, $\mathrm{n}^{\circ} 6,1^{\mathrm{er}}$ avril 1910, paraissent, sous le même titre, deux livraisons : «Comment on nous voit en France », ibid., vol. 5, n $\mathrm{n}^{\circ} 1,1^{\mathrm{er}}$ mai 1910, p. [9]-19, et Henry de Bruchard, «La France et la question canadienne », ibid., p. [20]-24 ; puis «Comment on nous voit en France » [suite], ibid., vol. 5, n 2, $1^{\text {er }}$ juin 1910, p. 104-110. 
Lequel n'exprimerait une admiration sans réserve pour nos compatriotes du Canada qui, dans le passé, ont défendu avec tant d'héroïsme notre drapeau, et qui, dans le présent, continuent une lutte pacifique aussi méritoire pour sauvegarder, sur notre ancienne possession, notre langue, notre littérature, notre religion, nos traditions, tout ce qui constitue notre patrimoine religieux, tout ce qui maintient le prestige de notre vieille France. Ah! certes, nous les aimons profondément ces Canadiens-français [sic], car ils ont conservé, plus que nous peut-être, la fidélité du souvenir, rendu plus vivace par les épreuves traversées. Et quand nous sommes enclins à quelque découragement, leur exemple nous est un réconfort ${ }^{100}$.

Un Frédéric Masson, membre de l'Académie, croit même expliquer cet état de fait :

J'ai la conviction que le développement considérable de notre race en terre américaine est dû à ce qu'elle a échappé aux révolutions qui ont déchiré la mère-patrie [...]. Il est des provinces françaises où la race fut vraiment française - je dis fut. [...] quand en France, il n'y aura plus de Français, c'est par la France [c]anadienne, qui, j'espère alors se sera libérée et affranchie, qu'on jugera la race, notre race vraie, la race de France $^{101}$.

Pour sa part, M. Barth, membre de l'Institut, exprime des sentiments mélangés : «Et en y pensant que pourrais-je éprouver, si ce n'est une profonde douleur de ce que mes ancêtres n'ont pas fait, et une plus grande admiration de ce qu'ont fait et continuent de faire les descendants des vôtres ${ }^{102}$. » Dans un article à part du même numéro ${ }^{103}$, mais beaucoup plus développé, Henry de Bruchard propose son interprétation monarchiste et conservatrice qui se change en attaque contre ses compatriotes :

Le principe de l'autorité, les bienfaits de l'hérédité monarchique, les règles civilisatrices du catholicisme, nos frères du

100. Henry Simond, «Comment on nous voit en France », ibid., vol. 5, n 1 , $1^{\text {er }}$ mai 1910 , p. 14.

101. Frédéric Masson, « Comment on nous voit en France », ibid., p. 16.

102. M. Barth, « Comment on nous voit en France », ibid., p. 17.

103. Henry de Bruchard, «La France et la question canadienne », ibid., p. [20]-24. 
Saint-Laurent en gardaient l'exercice et les bienfaits, avec une couronne étrangère, alors que nos mains sacrilèges ruinaient, par la base, le grand édifice protecteur, et s'efforçaient de construire, en commençant par la toiture, un nouveau bâtiment, dont les plans et les devis, dressés idéologiquement à Londres ou à Genève, ne convenaient ni à notre sol, ni aux matériaux qu'on en pouvait extraire, ni au climat de notre pays, ni à nos habitudes ancestrales ${ }^{104}$.

Dans le cahier suivant, après la phrase brève de Jules Lemaître qui dit éprouver « la plus profonde sympathie pour ces Français restés intacts ${ }^{105} »$, le comte Léonce de Larmandie prétend avoir « toujours aimé et admiré la France d'outre mer, qui a si bien conservé - beaucoup mieux hélas ! que nous-mêmes - nos vieilles traditions de foi et d'honneur ${ }^{106} »$. Toutefois, Salomon Reinach, un autre membre de l'Institut, remarque : « Mais de même que le langage des Franco-[C]anadiens est resté, par bien des traits, celui du siècle de Louis XIV, il me semble que la mentalité de nos concitoyens d'outre-mer n'a pas suivi, depuis le XVIII siècle, l'évolution de la pensée en France, évolution à la fois libérale et libératrice, que le monde admire, alors que souvent nous en médisons nous-mêmes ${ }^{107}$. »

Produites pour la circonstance par des admirateurs lointains qu'on a sollicités, ces approbations paraîtront plutôt complaisantes. Si ces personnalités font unanimement l'éloge du conservatisme, vite suivi d'un mea culpa d'occasion ou, parfois en sourdine, de la mention du retard qu'un tel état engendre, leurs déclarations auront moins de poids que celles qui proviennent d'un mouvement personnel et de témoins directs. Mais elles vont dans la même direction. L'opinion contemporaine d'un Brestois, formulée peu de temps après son arrivée dans la Vieille Capitale en octobre 1911, le confirme. À propos de « ces paysans opiniâtres, $[\ldots]$ restés là depuis deux cents ans », l'écrivain note :

104. Ibid., p. 22.

105. Jules Lemaître, « Comment on nous voit en France » [suite], p. 104.

106. Comte Léonce de Larmandie, « Comment on nous voit en France» [suite], ibid., p. 105.

107. Salomon Reinach, « Comment on nous voit en France » [suite], ibid., p. 108. 
C'est à peine s'ils ont modifié, pour se défendre contre le froid homicide, le costume traditionnel du pays d'où ils venaient ; tout le reste, langue, croyances, coutumes, ils l'ont gardé intact, sans arrogance, presque sans y songer, sur ce continent nouveau, au milieu de populations étrangères ; comme si un sentiment inné, naïf, et que d'aucuns jugeront incompréhensible, leur avait enseigné qu'altérer en quoi que ce fût ce qu'ils avaient emporté avec eux de France, et emprunter quoi que ce fût à une autre race, c'eût été déchoir un peu ${ }^{108}$.

Ces propos encore émus par la découverte de cette francophonie résistante, que Louis Hémon consigne au terme de son « Itinéraire de Liverpool à Québec », reviendront dans son roman Maria Chapdelaine et s'achèveront par la phrase célèbre : «Ces gens sont d'une race qui ne sait pas mourir ${ }^{109}$. »

Interlocuteurs plus nombreux peut-être, mais rien de nouveau dans ces discours du XIX $X^{\mathrm{e}}$ siècle et $\mathrm{du} \mathrm{XX}^{\mathrm{e}}$ commençant. Déjà au tournant du XVIII ${ }^{\mathrm{e}}$ siècle, Diéreville, dans la relation de son séjour à Port-Royal en Acadie (1699-1700), avait célébré en ses vers la remarquable résistance de cette population qui restait fidèle à la France en dépit des changements d'allégeance politique.

Disons encor plus à la gloire / De tous ces Habitans, ils l'ont bien mérité. / Ne finissons pas leur Histoire / Sans y mettre un beau trait de leur fidélité. [...] / Dans quel temps marquoientils avoir tant de constance? / Dans le temps même que la France/Ne pouvoit pas les soulager, /Et qu' on leur promettoit une entiere assistance, /S'ils avoient bien voulu changer. / Ils ne se laissoient point aller à cette amorce, / Ils ne vouloient point être Anglois, / Et de tout leur courage ils défendoient leurs droits ; / Contraints de ceder à la force, / Tous vaincus qu'ils étoient, ils demeuroient François. [...]/Que de Peuples réduits à leur extrêmité,/Pour être plus heureux auroient changé de Maître ! [...]/Je croy que pour leur Prince un amour pur

108. Louis Hémon, Écrits sur le Québec. Avant-propos et postface de Chantal Bouchard. Notes et variantes par Ghislaine Legendre et Chantal Bouchard, [Montréal], Éditions du Boréal, « Boréal compact classique » 52, [1993], « Itinéraire de Liverpool à Québec », p. 53.

109. Id., Maria Chapdelaine. Récit du Canada français, Présentation d'Aurélien Boivin. Postface de Bernard Clavel, Montréal, Bibliothèque québécoise, 1990, p. 194. 
\& tendre, / Sur l'attrait du profit l'a toûjours emporté : / Leur mérite est plus grand, \& je ne puis comprendre / Comment ils ont tant resisté ${ }^{110}$.

Des dizaines d'autres témoins, depuis le XVII ${ }^{e}$ siècle jusqu'à nos jours, tiennent semblable propos, louant tantôt la qualité d'un français sans accent, la richesse et l'ancienneté de son vocabulaire ou d'autres aspects de la vie populaire. Bien sûr, ces visiteurs voient, décrivent, exaltent ou condamnent les us et coutumes des Canadiens qu'ils rencontrent, suivant qu'ils utilisent la lorgnette du missionnaire, de l'explorateur, du bureaucrate, du commerçant, du naturaliste, du musicien, de l'écrivain ou du touriste. Pourtant, toutes leurs dépositions sont unanimes ${ }^{111}$. Selon Lothar Wolf, qui a scruté les documents en rapport avec la langue parlée et qui résume leurs perceptions,

[c]es témoignages paraissent [donc] crédibles et ils concordent tous : on parlait, en Nouvelle-France entre 1650 et 1760, une langue française qui n'avait rien à envier à celle de France, voire de Paris. On y parlait un français «pur», «pareil au nôtre », par référence au français de France. Les témoins n'y décèlent « aucun accent» ou constatent que l'accent des Canadiens « est aussi bon qu'à Paris ». Enfin, ils ne signalent aucun parler dialectal ou régional et attestent, au contraire, qu' « il n'y a pas de patois dans ce pays $»^{112}$.

110. Diéreville, «Relation des manieres tant des habitans que des sauvages de la Nouvelle France », dans Relation du voyage du Port Royal de l'Acadie, ou de la Nouvelle France suivie de Poésies diverses, Édition critique par Normand Doiron, Montréal, Les Presses de l'Université de Montréal, "Bibliothèque du Nouveau Monde », 1997, p. 216-218. Publié pour la première fois à Rouen en 1708, ce voyage a eu lieu du 20 août 1699 au 10 novembre 1700.

111. Voir le chapitre 2 « La Langue des premiers Canadiens » dans Le Français au Québec. 400 ans d'histoire et de vie, publication du Conseil supérieur de la langue française sous la direction de Michel Plourde avec la collaboration de Hélène Duval et de Pierre Georgeault, [Montréal], Éditions Fides, [2003], p. 25-42, notamment les textes de Lothar Wolf, de Raymond Mougeon et de Jean-Denis Gendron. Cf. la Bibliographie linguistique du Canada français de James Geddes et Adjutor Rivard (1906) continuée par Gaston Dulong, Québec, PuL, et Paris, Librairie C. Klincksieck, 1966, XXXII-167 p., ouvrage toujours utile pour les périodes considérées.

112. Lothar Wolf, "Les Colons de Nouvelle-France », dans Le Français au Québec, op. cit., p. 27. 
Des impressions de ces témoins, le linguiste Jean-Denis Gendron tirera quelques leçons : il notera bien « quelques divergences de vocabulaire " entre les deux parlers, mais il conclura que « l'ensemble des témoignages » sur la langue parlée en NouvelleFrance « montre bien que les fondements de la langue française au Canada se trouvent [...] avant tout dans la langue commune française de l'époque qui a tôt fait de prédominer et de l'emporter sur toutes les autres formes de parler, s'imposant ainsi précocement à l'ensemble des Canadiens ${ }^{113} »$. Selon lui, c'est le maintien de ces assises qui caractériserait le français québécois jusqu'à la Révolution tranquille des années 1960, car :

[...] à partir de la Révolution française (1789) et des bouleversements sociaux qu'elle engendre, le français de Paris va faire des choix de prononciation qui vont éloigner de façon sensible la forme soignée de ce parler du français québécois traditionnel, resté, quant à lui, à peu près stationnaire, figé dans la forme qu'il avait prise à la fin du Régime français, forme qui, avec quelques variations, était alors commune aux deux parlers ${ }^{114}$.

La déférence que les Français d'Europe affichent de la sorte, et dont les documents dépistés sur près de trois siècles témoignent abondamment, ne serait donc ni fantaisiste ni d'invention récente. Mais la multiplication de telles perceptions assurerait-elle pour autant que, plus qu'un cliché réchauffé, elle est porteuse d'une quelconque vérité ? Qu'est-ce qui motivait ces personnalités à tant vanter la résistance du patrimoine qu'ils découvraient chez les Canadiens français ? On peut comprendre que certains monarchistes aient eu intérêt à glorifier la France d'avant la Révolution dont ils surprenaient ici les reliquats ; que d'autres, émus de rencontrer au Canada les descendants des colons français partis de France au XVII et au XVIII ${ }^{\mathrm{e}}$ siècle, ou se culpabilisant de l'abandon de cette colonie par leur pays, aient idéalisé cette forme d'exotisme. Mais, même en admettant que des témoins plus tardifs

113. Jean-Denis Gendron, «Le Français des premiers Canadiens », dans Le Français au Québec, op. cit., p. 42.

114. Ibid., p. 41. 
aient connu et repris un discours déjà colporté par la rumeur, il serait bien difficile de déceler dans leurs rapports une source commune, telle que pourraient en fournir les souvenirs de voyage du siècle présent par exemple, où les voyageurs ont accès à des guides touristiques et à des agences avec lesquels ils planifient les éléments pittoresques à voir. Rien ne permet à ce stade-ci de présumer que ces témoins étaient de mauvaise foi, qu'ils répétaient des lieux communs et qu'ils n'ont pas vraiment vu ce qu'ils ont rapporté ${ }^{115}$.

\section{B. La fascination d'un folklore vivifié}

Dès lors, peut-on avec raison se demander si des chercheurs ont observé pareils comportements et ce qu'ils pensent de la qualité du transfert des traditions orales européennes chez les Français d'Amérique. Les avis de spécialistes, qui ont comme point de référence leurs recherches en France, s'accorderont-ils au concert élogieux des écrivains et autres visiteurs de passage ?

L'enquête de la Revue franco-américaine avait fini par atteindre un grand folkloriste français, Paul Sébillot, auteur de nombreux ouvrages sur les chansons et contes populaires de la Bretagne, qui ajouta sa réponse à celles de l'élite française. Il rappela sa correspondance avec Ernest Gagnon, l'auteur du "précieux recueil des Chansons populaires $d u$ Canada » dans lequel il avait « retrouvé nombre de thèmes familiers au pays malouin », et qu'il avait vainement tenté d'intéresser à la cueillette

115. On trouvera encore de nombreux témoignages élogieux dans la patiente compilation d'Armand Yon, Le Canada vu de France (1830-1914), Québec, Pul, « Vie des lettres québécoises »15, 1975, [8]-237 p. Tant dans les deux périodes proposées, «l'ère du sentiment (1830-1880)» et « une ère de critique (1880-1914)», le tableau que l'auteur brosse de l'opinion française sur le Canada reprend ce discours, les « témoins insist[ant] particulièrement sur la fidélité des Canadiens français aux traditions de la mère patrie » (p. 24). À partir des années 1880, « le peuple canadien sera passé au crible d'une critique fréquente, souvent injuste, parfois salutaire » (ibid.) ; essentiellement issue du clan laïc ou anticlérical, elle attaque, comme rétrograde et faisant obstacle au développement du pays, l'omniprésence du clergé, dont les méthodes d'enseignement sont jugées désuètes ou retardataires. 
des contes populaires ${ }^{116}$. À l'occasion du Congrès international des traditions populaires, qu'il organisa à Paris en 1900, il avait fait la connaissance d'Honoré Beaugrand, qui lui avait remis un exemplaire de son recueil La Chasse-galerie ${ }^{117}$. Il 1'aurait apparemment convaincu de s'intéresser au conte populaire, mais « seule sa mort prématurée » l'en aurait empêché118.

Je lui disais que j'étais persuadé que dans votre pays, si conservateur de ce qui vient de la mère-patrie [sic], les anciens contes n'étaient pas oubliés, et qu'un explorateur diligent pourrait y retrouver les éléments d'un volume de contes populaires qui serait le digne pendant des chansons d'Ernest Gagnon. Les contes auraient d'autant plus d'intérêt qu'ils présenteraient vraisemblablement à peu près la forme qu'ils avaient lorsqu'au XVII ${ }^{\mathrm{e}}$ et au XVIII ${ }^{\mathrm{e}}$ siècle, ils sont venus de l'ancien pays ${ }^{119}$.

Selon Luc Lacourcière, une telle hypothèse, due au « flair des folkloristes étrangers » comme Sébillot qui n'est pourtant jamais venu au Canada, aboutira après quelques détours et un peu de retard aux premières enquêtes de Marius Barbeau sur le conte populaire $^{120}$.

Jean-Jacques Ampère, Théodore de Puymaigre, Julien Tiersot, qui étaient contemporains de Sébillot, puis André Varagnac, René Maunier, Paul Delarue dans la première moitié du $\mathrm{XX}^{\mathrm{e}}$ siècle furent littéralement séduits par la survivance du patrimoine oral au Canada français, de la chanson folklorique notamment ${ }^{121}$. Les

116. C'est Luc Lacourcière qui signale ce détail et cette publication dans « Contes et légendes ", Congrès de la refrancisation, Québec 21-24 juin 1957, Québec, Les Éditions Ferland, 1959, vol. vi, p. 25-35.

117. H[onoré] Beaugrand, La Chasse[-]galerie. Légendes canadiennes, [Illustrations d'Henri Julien], Montréal, [s. é.], 1900, 123 p.

118. Paul Sébillot a recensé le livre de Beaugrand dans la Revue des traditions populaires, Paris, 1900, p. 49.

119. Paul Sébillot, «Comment on nous voit en France », La Revue francoaméricaine, vol. $5, \mathrm{n}^{\circ} 2,1^{\mathrm{er}}$ juin 1910 , p. 110.

120. Luc Lacourcière, "Contes et légendes ", op. cit., p. 26.

121. Nous les citons dans notre article «La Découverte de la chanson traditionnelle française en Canada ou la fascination d'un folklore vivifié », dans $L a$ Bretagne et la littérature orale en Europe, Textes réunis et publiés par Fañch Postic, Mellac-Brest, Centre de recherche bretonne et celtique, Centre de recherche et de documentation sur la littérature orale, Centre international de rencontre des cultures de tradition orale, 1999, p. 261-289. 
points de vue de quelques autres spécialistes français de la seconde moitié du siècle, ethnomusicologues et ethnolinguistes, ajouteront des avis éclairés qui conforteront ces impressions.

En 1956, paraissait une tranche substantielle de la collection lyrique de Marius Barbeau, Chansons folkloriques françaises au Canada par Marguerite et Raoul d'Harcourt ${ }^{122}$. D'entrée de jeu, le « prélude aux chansons » du professeur d'analyse musicale au Conservatoire de Paris, le compositeur Olivier Messiaen, reconnaissait que « [1] a différence entre chants français de France et chants français du Canada réside surtout dans la musique » en précisant que « [1] a survivance des modes grégoriens est plus nette au Canada que chez nous ${ }^{123} »$. Puis, en introduction, le couple d'ethnomusicologues français explique les circonstances qui les amenèrent à l'examen d'un choix des 1000 premières chansons recueillies entre 1916 et 1918 pour le compte du Musée national du Canada : «C'est en prenant conscience de l'exceptionnelle survivance de la chanson française passée outre-Atlantique à un moment où son épanouissement durait encore, que nous avons trouvé la raison déterminante de cette étude ». Il n'est pas vain de rappeler que Marguerite Béclard d'Harcourt avait préfacé vingt ans plus tôt le Romancero du Canada de Marius Barbeau, où son émerveillement, qui s'avéra durable, s'était manifesté une première fois ${ }^{124}$. Le présent échantillonnage leur fournissait un matériau inespéré, car « le prestige des mélodies françaises au Canada, dont le lustre n'est pas éteint, subsistait intact au début de ce siècle ${ }^{125} »$. En signalant le haut calibre des chanteurs, ces « excellents informateurs canadiens dont nous avons noté le

122. Marguerite et Raoul d'Harcourt, Chansons folkloriques françaises au Canada, op. cit., 1956, XII-449 p. L'étude porte sur 240 chansons de la collection de Marius Barbeau.

123. Ibid., p. [x].

124. Marius Barbeau, Romancero du Canada, [Montréal], Éditions Beauchemin ; [Toronto, Macmillan], 1937, 254 p. Préface de Marguerite Béclard d'Harcourt. Voir aussi notre article «Le Chercheur de trésors ou l'influence d'un livre. Marius Barbeau et le Romancero du Canada », CC, n 7, 2006, p. 105.

125. M. et R. d'Harcourt, Chansons folkloriques françaises au Canada, op. cit., p. 3. 
répertoire ${ }^{126} »$, et l'étendue de leur catalogue, soit 200 chansons pour François Saint-Laurent et 350 pour Vincent-Ferrier de Repentigny, ils s'interrogent : « Où donc trouverait-on aujourd'hui en France de tels informateurs ${ }^{127}$ ? »

En plus de l' « exceptionnelle survivance » du fonds français au Canada, de l'abondance du répertoire et de la qualité des interprètes populaires, les auteurs du chansonnier estiment le plan musical supérieur parce que restituant des caractères plus anciens et à l'abri des courants scolastiques : ils notent des « lignes mélodiques souvent plus généreuses, plus développées [et des rythmes plus subtils] que celles auxquelles nous étions accoutumés », que le mode d'ut "garde volontiers une forme archaïque et il laisse une plus large place que chez nous aux modes anciens qui restent bien vivants », ce qui les porte à « penser qu'à travers ces versions canadiennes on puisse atteindre la musique de nos chansons, plus sûrement que dans bien des transcriptions tardives, faites en France ${ }^{128} \gg$. Plus encore, l'esthétique est particulièrement remarquable : «Le style lyrique très orné, qui atteint ici une grande beauté, semble à peu près perdu en France. » S'ils corroborent chez eux « la perte du vrai style fleuri », ils jugent que « [1] es chanteurs canadiens ont dû en hériter ; ils l'ont conservé par goût, renchérissant même sur cette tendance, comme François Saint-Laurent et Vincent-Ferrier de Repentigny ${ }^{129} »$. Enfin, les auteurs versent d'autres points au dossier : la survivance « des rythmes vraiment libres », " qui rend l'apport du Canada si précieux pour l'histoire de la musique populaire française ${ }^{130} »$, et la floraison de « refrains omnibus » qui peuvent s'adapter à plusieurs chansons ${ }^{131}$.

Au terme de la longue analyse des d'Harcourt, qu'en sa qualité d'ethnographe il suivit certainement avec grand intérêt, Marius

126. Ibid., p. 22.

127. Ibid., p. [11].

128. Ibid., p. 6.

129. Ibid., p. 43-44.

130. Ibid., p. 48.

131. Ibid., p. 52. 
Barbeau tira aisément quelques conclusions essentielles du travail minutieux des ethnomusicologues français. On les trouve dans sa préface où il affirme sans ambages : d'abord, que « [1]es chansons de l'Amérique française sont pour la plupart représentatives du répertoire de la vieille France » à l'époque où « la musique populaire, de tradition purement orale, conservait encore ses traits archaïques et moyenâgeux »; ensuite, que « [p]eut-être ces versions furent-elles plus fidèlement conservées au Canada, loin de la mère patrie, grâce à un isolement de longue durée, que les variantes françaises ultérieures dont beaucoup ont subi les retouches des lettrés »; et qu' «[e]n somme, l'art musical et poétique de la chanson populaire française s'est mieux et plus abondamment conservé sur les rives jadis fleurdelisées du SaintLaurent qu'il ne l'a été à ses sources provinciales françaises, celles-ci de tout temps soumises à des influences rénovatrices ». Et il clôt le débat : « En eussiez-vous douté que la présente étude vous en fournirait la preuve convaincante ${ }^{132}$. »

Pour sa part, l'ethnolinguiste Geneviève Massignon s'était intéressée au «problème de la survivance d'un parler français transplanté depuis le XVII ${ }^{\mathrm{e}}$ siècle » dans une étude marquante sur les diverses régions de l'Acadie « où survit le parler le plus archaïque ». Son investigation, menée principalement en 1946, « fut partout accompagnée d'une enquête sur la littérature orale, dont [elle] avai[t] entrevu la richesse chez un de [s]es informateurs du Madawaska ». Si elle se proposait de retracer « les origines provinciales françaises de ce petit peuple », elle étudia aussi « sa faculté d'adaptation au Nouveau Monde, aussi intéressante, du point de vue sémantique, que son conservatisme évident ${ }^{133} \gg$. En 1994, on publiait à titre posthume sa thèse complémentaire consacrée spécialement à la chanson populaire, restée inédite depuis 1962. Commentant en introduction l'état de la tradition qu'elle avait explorée, elle observait : « La richesse des traditions

132. «Préface », ibid., p. IX-X.

133. Geneviève Massignon, Les Parlers français d'Acadie. Enquête linguistique, Paris, Librairie C. Klincksieck, [1962], vol. I, p. 10-11. 
populaires en Acadie est ce qui frappe d'abord le chercheur : que de gens y connaissent des chansons, combien se plaisent à les écouter, et comme elles sont variées !»Et aussitôt, elle renchérissait : «Plus remarquable encore que leur foisonnement est l'étonnante fraîcheur de celles-ci, émigrées depuis tant d'années. » Elle comparait alors l'Acadie à un autre peuple qui avait conservé son folklore oral « avec une pureté et une fidélité qu'on n'a pas toujours rencontrées dans la patrie d'origine de cette tradition » pour conclure : " On pourrait dire de même à propos du folklore canadien et acadien ${ }^{134}$. »

Invité plus récemment à commenter la «place du conte populaire français d'Amérique dans la tradition orale de la francophonie ${ }^{135} »$, un ethnologue brestois l'appréciait ainsi : « je ne suis pas éloigné de penser que dans ce sens [en termes de qualité, de vitalité], et pour ce qui concerne son état de santé, il occupe la première place. » Justifiant sa position, Donatien Laurent ajoutait que « les nombreuses publications faites ici depuis plus d'un siècle [...] attestent de la façon la plus éclatante la vitalité des recherches menées au Canada français sur un terrain qui me paraît, pour des raisons diverses, avoir beaucoup mieux résisté que sur le continent à la déstructuration du milieu traditionnel ${ }^{136} »$. S'interrogeant ensuite sur la façon dont cette «branche détachée du tronc continental» avait évolué en sol américain, il retenait qu'elle « représente une sorte de laboratoire, de champ d'expérience et d'observation incomparable pour l'étude du conte populaire français de tradition orale et du fonctionnement de cette tradition », d'autant précieuse qu'elle « nous restitue un milieu qui chez nous, en France, a disparu presque partout depuis près d'un siècle : un véritable milieu de

134. Geneviève Massignon, Trésors de la chanson populaire française. Autour de 50 chansons recueillies en Acadie, I. Textes, Revu, corrigé et édité par Georges Delarue, [Paris], Bibliothèque nationale de France, 1994], p. XLII.

135. Donatien Laurent, « La Place du conte populaire français d'Amérique dans la tradition orale de la francophonie », dans J.-P. Pichette, L'Euvre de Germain Lemieux, s.j. Bilan de l'ethnologie en Ontario français, Sudbury, Prise de parole et Centre francoontarien de folklore, « Ancrage », 1993, p. 275-285.

136. Ibid., p. 276. 
tradition orale $\mathrm{e}^{137} »$. Finalement, il considérait le conte français d'ici comme « une algue qui se déploie dans la mer comparée à l'algue séchée, conservée dans un herbier. Nous, nous n'avons plus, dans les meilleurs cas, que l'herbier. Vous, vous avez encore la possibilité d'observer et de rendre compte de la vie de cette $\operatorname{tradition}^{138} »$. Ce faisant, il validait l'opinion que Paul Delarue avait confiée à Luc Lacourcière en 1954 : « Vous savez que nous ne sommes, par le monde, qu'un nombre assez réduit de spécialistes à nous intéresser au conte populaire et vous êtes peutêtre le seul à travailler sur une matière encore vivante $[\ldots]^{139}$. "

Ces commentaires inattendus coïncidaient avec les résultats de notre étude comparative fraîchement publiée, notamment sur la continuité exemplaire de la tradition du conte populaire au Canada français. Comme la demi-douzaine de comparatistes de 1'Université Laval l'avaient, à leur façon, fait avant nous ${ }^{140}$, nous y soulignions, entre autres, que la tradition du Nouveau Monde n'avait, sur ce plan, rien à envier à celle de l'Ancien, ni pour le nombre des versions ni pour leur qualité, à un point tel que notre volumineuse étude confirmait, elle aussi, « la vigueur de la tradition française transplantée au Canada, plus riche et mieux conservée ici que dans son pays d'origine » tout en demeurant « très fidèle à ses sources européennes ${ }^{141}$ ». Autre point de jonction,

137. Ibid., p. 278.

138. Ibid., p. 279.

139. Archives de folklore et d'ethnologie de l'Université Laval, Fonds LucLacourcière (P 178), Paul Delarue à Luc Lacourcière, lettre du 11 septembre 1954.

140. Dans des mémoires de maîtrise et des thèses de doctorat dirigés par Luc Lacourcière : Hélène Bernier, La Fille aux mains coupées (conte-type 706), Québec, Pul, « Archives de folklore » [« AF »] 12, 1971, XII-191 p. ; Germain Lemieux, PlacideEustache. Sources et parallèles du conte-type 938, Québec, PUL, « AF » 10, 1970, VIII214 p. ; Nancy Schmitz, La Mensongère (conte-type 710), Québec, Pul, " Af » 14, 1972, XIV-310 p. ; Pauline Éthier, «Monographie du conte-type 590 A, L’Épouse traîtresse dans la tradition canadienne-française », Québec, Université Laval, thèse de maîtrise ès arts, 1976, XIV-246 p. ; Margaret Low, «L'Oiseau mystérieux du château volant : monographie internationale du conte-type 708 A (462)», Québec, Université Laval, thèse de Ph.D., juin 1978, Xxvi-473 p.

141. J.-P. Pichette, L'Observance des conseils du maître. Monographie internationale du conte type A.-T. 910 B précédée d'une introduction au cycle des Bons conseils (A.T. 910-915), Québec, PUL, « AF » 25 ; Helsinki, Academia Scientiarum Fennica, «Folklore Fellows Communications » 250, 1991 (xx-672 p.), p. 570-571. 
nos deux positions affichaient une commune prudence dans l'attribution d'une origine irlandaise à certains de nos contes populaires introuvables en France, hypothèse séduisante qui obnubilait parfois quelques étudiants. Constatant que les traditions bretonnes et franco-ontariennes avaient puisé «pour une large part [leurs] racines dans un terreau commun : l'Ouest de la France, voire même la Haute-Bretagne », Donatien Laurent concluait par cette remarque finale :

Quant au caractère « celtique », que Paul Delarue reconnaissait au conte canadien et dont il voyait - peut-être un peu rapidement - l'explication dans un apport irlandais récent, il est également présent en Basse Bretagne - ne serait-ce qu'à travers la langue - et constitue lui aussi un trait d'union entre nos deux répertoires narratifs traditionnels qu'il conviendrait sans doute de mieux préciser $^{142}$.

Cette ouverture à la collaboration déboucha sur notre intervention au colloque international La Bretagne et la littérature orale en Europe qui commémorait à Quimperlé, en 1995, le centenaire de la mort de la Villemarqué143 : la découverte du domaine de la chanson, sujet dicté par la circonstance, entraîna pareils résultats : soit la fascination des visiteurs et des chercheurs européens, français surtout, devant la haute qualité et la vitalité de la chanson folklorique française en Amérique, alors qu'à leurs yeux elle s'était anémiée en Europe ${ }^{144}$. Cela, Donatien Laurent le certifiera lui-même une fois encore dix ans plus tard dans l'examen d'une chanson historique destiné aux Mélanges Dupont : « Mais si l'on se bornait, pour en faire l'étude, à ne prendre en compte que les versions collectées en France, pays pourtant directement concerné par l'événement, on serait bien en peine. C'est l'apport des

142. Donatien Laurent, « La Place du conte [...]», op. cit., p. 285.

143. Théodore Hersart de la Villemarqué (1815-1895), auteur d'un ouvrage fondateur, Barzaz-Breiz, chants populaires de la Bretagne (1839).

144. J.-P. Pichette, «La Découverte de la chanson traditionnelle française en Canada ou la fascination d'un folklore vivifié », op. cit. 
versions franco-américaines qui donne tout son poids et sa richesse à ce dossier ${ }^{145}$. »

Tous ces propos convergent et établissent que l'Amérique contribue, par la persistance qu'elle affiche, à mieux faire connaître l'étendue et la richesse du patrimoine oral français à la France elle-même. Ainsi, se vérifient la conviction d'Hubert La Rue concluant son étude comparative d'une chanson populaire, « que presque toujours l'avantage reste tout entier à nos chansons canadiennes $^{146} »$; tout autant que l'étonnement de l'auteur du Romancero du Canada confronté régulièrement au « silence de la documentation française ${ }^{147} »$, signe d'une érosion de la chanson traditionnelle en France ; et enfin 1'aveu de sa préfacière Marguerite Béclard d'Harcourt posant que « l'intérêt supérieur des versions canadiennes réside précisément en ce fait qu'elles nous permettent de compléter la physionomie du chant français en lui restituant quelques-uns de ses plus beaux traits ${ }^{148} »$, ce qu'elle justifiait par « une tradition plus fidèle que celle de leur pays d'origine ${ }^{149} »$.

\section{REMERCIEMENTS}

L'auteur adresse ses remerciements à ses collègues de la Société Charlevoix, qui ont lu et commenté copieusement deux versions de cet article, les membres du Groupe de recherche en études acadiennes et des Rencontres britto-franco-canadiennes qui ont tenu des journées d'étude et organisé un colloque international sur ce thème, ainsi que ceux qui lui ont fourni des renseignements ponctuels et dont les noms figurent dans les notes.

145. Donatien Laurent, « La Reddition du Foudroyant en 1758. Un épisode naval de la guerre de Sept Ans à travers la chanson française de tradition orale en France et en Nouvelle-France ", dans Entre Beauce et Acadie. Facettes d'un parcours ethnologique. Études offertes au professeur Jean-Claude Dupont. Textes réunis par J.-P. Pichette avec la collaboration de Jocelyne Mathieu, Richard Dubé et Yves Bergeron, [Québec], PUL, [2001], p. 260.

146. Hubert La Rue, "Les Chansons populaires et historiques du Canada ", op. cit., p. 328-329.

147. Marius Barbeau, Romancero du Canada, op. cit., p. 17.

148. «Préface », ibid., p. 11.

149. Ibid., p. [7]. 